\title{
The Penn State - Toruń Centre for Astronomy Planet Search stars $\star, \star \star$
}

\section{Lithium abundance analysis of the red giant clump sample}

\author{
M. Adamów ${ }^{1}$, A. Niedzielski ${ }^{1}$, E. Villaver ${ }^{2}$, A. Wolszczan ${ }^{3,4}$, and G. Nowak ${ }^{5,6,1}$ \\ 1 Toruń Centre for Astronomy, Faculty of Physics, Astronomy and Informatics, Nicolaus Copernicus University, Grudziadzka 5, \\ 87-100 Toruń, Poland \\ e-mail: [adamow; aniedzi; grzenow]@astri.umk.pl \\ 2 Departamento de Física Teórica, Universidad Autónoma de Madrid, Cantoblanco 28049 Madrid, Spain \\ e-mail: Eva.Villaver@uam.es \\ 3 Department of Astronomy and Astrophysics, Pennsylvania State University, 525 Davey Laboratory, University Park, PA 16802, \\ USA \\ e-mail: alex@astro.psu.edu \\ ${ }^{4}$ Center for Exoplanets and Habitable Worlds, Pennsylvania State University, 525 Davey Laboratory, University Park, PA 16802, \\ USA \\ 5 Instituto de Astrofísica de Canarias, C/ vía Láctea, s/n, 38205 La Laguna, Tenerife, Spain \\ 6 Departamento de Astrofísica, Universidad de La Laguna, Av. Astrofísico Francisco Sánchez, s/n, 38206 La Laguna, Tenerife, Spain \\ Received 10 January 2014 / Accepted 30 June 2014
}

\begin{abstract}
Context. Standard stellar evolution theory does not predict existence of Li-rich giant stars. Several mechanisms for Li-enrichment have been proposed to operate at certain locations inside some stars. The actual mechanism operating in real stars is still unknown. Aims. Using the sample of 348 stars from the Penn State - Torun Centre for Astronomy Planet Search, for which uniformly determined atmospheric parameters are available, with chemical abundances and rotational velocities presented here, we investigate various channels of Li enrichment in giants. We also study Li-overabundant giants in more detail in search for origin of their peculiarities. Methods. Our work is based on the Hobby-Eberly Telescope spectra obtained with the High Resolution Spectrograph, which we use for determination of abundances and rotational velocities. The $\mathrm{Li}$ abundance was determined from the ${ }^{7} \mathrm{Li} \lambda 670.8 \mathrm{~nm}$ line, while we use a more extended set of lines for $\alpha$-elements abundances. In a series of Kolmogorov-Smirnov tests, we compare Li-overabundant giants with other stars in the sample. We also use available IR photometric and kinematical data in search for evidence of mass-loss. We investigate properties of the most Li-abundant giants in more detail by using multi-epoch precise radial velocities.

Results. We present $\mathrm{Li}$ and $\alpha$-elements abundances, as well as rotational velocities for 348 stars. We detected Li in 92 stars, of which 82 are giants. Eleven of them show significant Li abundance $A(\mathrm{Li})_{\mathrm{NLTE}}>1.4$ and seven of them are Li-overabundant objects, according to common criterion of $A(\mathrm{Li})>1.5$ and their location on HR diagram, including TYC 0684-00553-1 and TYC 3105-001521, which are two giants with $\mathrm{Li}$ abundances close to meteoritic level. For another 271 stars, upper limits of Li abundance are presented. We confirmed three objects with increased stellar rotation. We show that Li-overabundant giants are among the most massive stars from our sample and show larger than average effective temperatures. They are indistinguishable from the complete sample in terms of their distribution of luminosity, metallicity, rotational velocities, and $\alpha$-elements abundances. Our results do not point out to one specific Li-enrichment mechanism operating in our sample of giants. On the contrary, in some cases, we cannot identify fingerprints of any of known scenarios. We show, however, that the four most Li-rich giants in our sample either have low-mass companions or have radial velocity variations at the level of $\sim 100 \mathrm{~m} \mathrm{~s}^{-1}$, which strongly suggests that the presence of companions is an important factor in the Li-enrichment processes in giants.
\end{abstract}

Key words. stars: fundamental parameters - stars: atmospheres - stars: late-type - techniques: spectroscopic - planetary systems

\footnotetext{
* Based on observations obtained with the Hobby-Eberly Telescope, which is a joint project of the University of Texas at Austin, the Pennsylvania State University, Stanford University, LudwigMaximilians-Universität München, and Georg-August-Universität Göttingen. Based on observations made with the Italian Telescopio Nazionale Galileo (TNG) operated on the island of La Palma by the Fundación Galileo Galilei of the INAF (Istituto Nazionale di Astrofisica) at the Spanish Observatorio del Roque de los Muchachos of the Instituto de Astrofísica de Canarias. Based on observations made with the Nordic Optical Telescope, operated on the island of La Palma jointly by Denmark, Finland, Iceland, Norway, and Sweden, in the Spanish Observatorio del Roque de los Muchachos of the Instituto de Astrofísica de Canarias. Based on observations
}

\section{Introduction}

Lithium, one of the three elements synthesized during the Big Bang nucleosynthesis, is easily destroyed in the stellar interiors

obtained with the HERMES spectrograph, which is supported by the Fund for Scientific Research of Flanders (FWO), Belgium, the Research Council of K.U.Leuven, Belgium, the Fonds National Recherches Scientific (FNRS), Belgium, the Royal Observatory of Belgium, the Observatoire de Genéve, Switzerland and the Thüringer Landessternwarte Tautenburg, Germany.

$\star \star$ Table 5 is only available at the CDS via anonymous ftp to cdsarc.u-strasbg.fr (130.79.128.5) or via http://cdsarc.u-strasbg.fr/viz-bin/qcat?J/A+A/569/A55 
at temperatures exceeding $\approx 2.5 \times 10^{6} \mathrm{~K}$. Abundance of $A(\mathrm{Li})^{1}=$ 3.3 dex, which represents the value found in the Solar System meteorites (Asplund et al. 2009), is considered the reference limit for population I stars.

The abundance of lithium, which is in the formation of a star, is expected to be preserved in the outermost surface layers of main sequence (MS) stars. As soon as the star evolves into the red giant branch (RGB) phase, the deepening of the convective zone brings material that has been exposed to high temperatures in the stellar interior to the stellar surface. Products of H-burning enrich the external layers with nitrogen and helium and the abundance of lithium is expected to drop to $A(\mathrm{Li})=1.5 \mathrm{dex}$, assuming that the star left the MS with a Li abundance close to 3.3 dex (Iben 1967). However, most observations of FGK dwarf stars reveal lithium abundances below the meteoritic reference value (e.g. Ghezzi et al. 2010; Ramírez et al. 2012), which indicates the existence of non-standard mixing mechanisms operating during the MS phase. This is probably the most important reason why the overwhelming majority of giant stars present lithium abundances below the 1.5 dex value predicted by the standard evolution theory (Iben 1967).

There are not many processes by which lithium can be produced in stars. However, 1-2\% of all observed giants (see e.g. Kumar et al. 2011; Lebzelter et al. 2012, and references therein) are lithium rich (stars with $A(\mathrm{Li})>1.5 \mathrm{dex})$. The abundance of lithium in such Li-rich giant stars can even exceed the amount of lithium found in meteorites.

The Cameron-Fowler (CF) mechanism (Cameron \& Fowler 1971 ) is usually invoked to explain the lithium overabundance in giant stars. Lithium production through this process involves, firstly, transport of ${ }^{3} \mathrm{He}$ from the outer layers to regions that are hot enough to ignite the ${ }^{3} \mathrm{He}(\alpha, \gamma){ }^{7} \mathrm{Be}$ burning reaction, and, secondly, transport of beryllium to the outer layers, where it decays to lithium, according to the reaction ${ }^{7} \mathrm{Be}\left(\mathrm{e}^{-}, v\right)^{7} \mathrm{Li}$. The CF mechanism is known to operate in stars with masses $4-8 M_{\odot}$ (Charbonnel \& Balachandran 2000) during their evolution on the asymptotic giant branch (AGB) in which deeper convection reaches the layers of nuclear burning (hot bottom burning).

The lower mass giants lack an additional mixing mechanism rather than convection itself to initiate the $\mathrm{CF}$ process, since the convection zone is too shallow there to feed ${ }^{3} \mathrm{He}$ to the hotter stellar interior.

Extra-mixing is expected to occur at a specific location on the Hertzsprung-Russell (HR) diagram, the luminosity function bump (LFB), which is associated with the removal of the molecular discontinuity that stems from the dredge-up processes. Charbonnel \& Balachandran (2000) and Kumar et al. (2011) have claimed that Li-rich giants concentrate in a narrow luminosity range associated with the LFB on the red giant branch and/or the red giant clump (RGC) region ${ }^{2}$, while other authors show that Li-rich giants can be found almost anywhere along the RGB (see e.g. Anthony-Twarog et al. 2013; Martell \& Shetrone 2013; Lebzelter et al. 2012). The Li enhancement mechanism on the RGB is thus still not clear and other processes explored to explain extra-mixing in RGB stars do not seem to be efficient enough. Thermohaline convection (Charbonnel \& Zahn 2007) seems too slow to be responsible for $\mathrm{Li}$ overabundance in giants, and magneto-thermohaline mixing (Denissenkov et al. 2009), and magnetic buoyancy (Busso et al. 2007) cannot explain observed values when $A(\mathrm{Li})>2.5$ dex.

\footnotetext{
$A(\mathrm{Li})=\log \frac{n(\mathrm{Li})}{n(\mathrm{H})}+12$.

2 Formed by stars in the stage of central helium burning (Cannon 1970).
}

External pollution offers an alternative to the CF production of lithium by a mechanism in which the chemical composition of the stellar photosphere might be altered through accretion of external material from type II supernova (SN) explosions (Woosley \& Weaver 1995), planets (Alexander 1967; Siess \& Livio 1999), or from a more evolved stellar companion (Sackmann \& Boothroyd 1999), such as an AGB star producing Li via $\mathrm{CF}$ mechanism. Each of these processes should present additional chemical features, besides the $\mathrm{Li}$ abundances, associated with them, that is, type II supernovae pollution should show increased levels of $\alpha$-elements (see e.g. Woosley \& Weaver 1995), while a stellar companion should display CNO cycle by-products. Planet engulfment might increase stellar rotation (Carlberg et al. 2010, 2012) that triggers internal lithium production (Siess \& Livio 1999) and/or could be connected with an increased mass loss that could be observable as an infrared excess (de La Reza et al. 1997) or as an additional component in the spectra of sodium lines (i.e. Drake et al. 2002).

There is still less than a hundred Li-overabundant giants known. As only a small percent of giants reveal that characteristic, this type of star is usually discovered in large spectroscopic surveys. Studying Li abundance for giants that are targets of a planet search gives unique opportunity to investigate $\mathrm{Li}$ abundance in connection with enhancement scenarios that involves a presence of stellar companions.

In this paper, we report on a high-resolution spectroscopic search for Li enrichment in giant stars located along the RGB. We use the extensive collection of high resolution spectra obtained for the Penn State-Torun Centre for Astronomy Planet Search (PTPS) program (Niedzielski et al. 2007; Niedzielski \& Wolszczan 2008). The full PTPS sample is composed of over 1000 evolved solar-type and intermediate-mass stars: giants, including the RGC stars (Zieliński et al. 2012), subgiants (Niedzielski et al., in prep.), and evolved dwarfs (Deka et al., in prep.). We have selected the most evolved subsample of PTPS stars, for which uniformly determined atmospheric and integrated stellar parameters are available from Zieliński et al. (2012). Those are 348 stars (343 giants and five dwarfs), for which we determine lithium abundances. We aim to study various channels of Li-enhancement in giants and their connection to the stellar parameters in an attempt to better constrain the characteristics of the enrichment mechanism.

The paper is organized as follows: in Sect. 2, we describe the observational material that we collected for the analysis. In Sect. 3, we describe the methods that we applied to our sample for $A(\mathrm{Li})$ and for the determination of $\mathrm{Al}, \mathrm{O}, \mathrm{Mg}, \mathrm{Ti}$, and $\mathrm{Ca}$ abundances. We also present rotational velocities obtained with two independent methods and discuss uncertainties of results and abundances determined for Arcturus. In Sect. 4, we present results of $A(\mathrm{Li})$ determinations and investigate possible links between various stellar parameters and lithium abundance. In Sect. 5, we discuss individual objects with enhanced Li abundances in more detail, and in Sect. 6, we present discussion of the results.

\section{Sample, observations and data reduction}

\subsection{Sample}

The sample presented in this paper contains 343 giants and five dwarfs out of the total $\sim 1000$ stars sample monitored in the PTPS project with the Hobby-Eberly Telescope (HET) for radial velocity variations using the high-precision iodine-cell technique. The total PTPS sample is mainly composed of evolved 


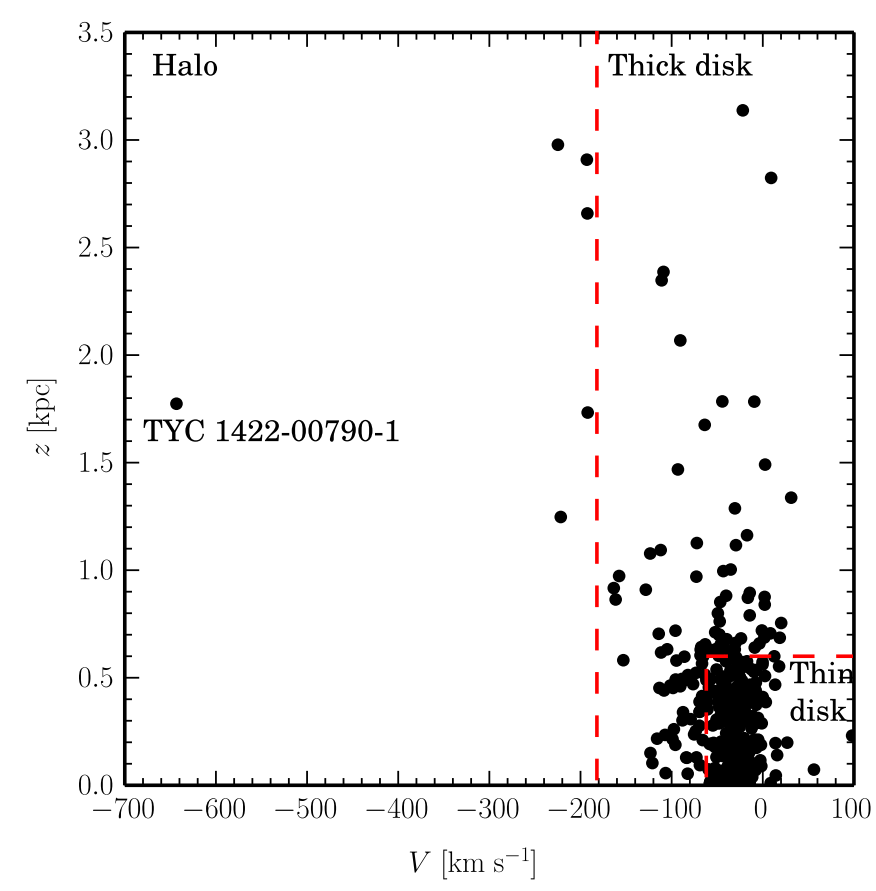

Fig. 1. Galactic rotational velocities of stars with respect to the Galactic Center $V$ versus distances from the galactic plane for stars analyzed in this paper. We mark the location of the different galactic components, thin disk, thick disk, and halo according to the Ibukiyama \& Arimoto (2002) criteria. The object TYC 1422-00790-1 is among the six halo stars present in the sample.

low-mass and intermediate-mass stars: $\sim 575$ giants, $\sim 225$ subgiants, and $\sim 200$ slightly evolved dwarfs. We have selected 343 giant stars and five dwarfs for which Zieliński et al. (2012) has already derived effective temperatures, $\log g$, turbulence velocities and metallicities through a purely spectroscopic analysis for this paper. Stellar luminosities, masses, and ages for those stars have been derived using fits to evolutionary tracks, and absolute radial velocities (RV) have been obtained with the crosscorrelation technique (see Zieliński et al. 2012).

A detailed analysis revealed that the sample studied, presumably consisting of RGC stars, is actually composed mainly of regular giant stars evolving along the RGB $(62 \pm 3 \%)$. Only $37 \pm 3 \%$ of giants (126 stars) belong to the RGC, according to the extended criteria in luminosity, which is widened to account for uncertainties, as presented by Zieliński et al. (2012); that is i.e. $4700 \mathrm{~K} \leq T_{\text {eff }} \leq 5100 \mathrm{~K}$ and $1.3 \leq \log L / L_{\odot} \leq 2$ (see Jimenez et al. 1998; Tautvaisiene \& Puzeras 2009). The sample also contains five dwarfs $(1.4 \pm 1 \%)$.

All stars have stellar parameters derived in a homogeneous manner from the same set of observations and instrument configuration. The stars have been shown not to be located in any particular region of the HR diagram which allows tests of the putative relation of $\mathrm{Li}$ overabundance and stellar evolution. Furthermore, all the stars included have reached the bottom of the RGB phase with the exception of two stars that are still in the subgiant phase and the five dwarfs mentioned previously.

To check whether we have a mix of stellar populations in the sample, we have adopted the Ibukiyama \& Arimoto (2002) criteria and obtained the location of the stars in the galactic height versus galactic rotational velocity plane as shown in Fig. 1. Distances from the galactic plane $\left(z_{\max }\right)$ have been determined using parallaxes from van Leeuwen (2007), which are available for $23 \%$ of stars. For the remaining $77 \%$ of objects, we have estimated distances using absolute magnitudes of Zieliński et al. (2012). Assuming an average uncertainty in the determinations of $M_{V}$ given in that paper, the uncertainty for the estimated distances is $\sim 100$ pc. We computed space velocity components $(U, V, W)$ following Johnson \& Soderblom (1987) but used the J2000 epoch in the coordinates and the transformation matrix, stellar distances, and proper motions from the Tycho/HIPPARCos Catalog (van Leeuwen 2007), and absolute RVs from Zieliński et al. $\left(2012 ; \sigma R V_{\mathrm{CCF}}=0.041 \mathrm{~km} \mathrm{~s}^{-1}\right)$. We then classified the stars into stellar populations using the rotation velocity component in the Galaxy $(V)$ and the distances from the galactic plane (see Ibukiyama \& Arimoto 2002).

We found (see Fig. 1) that most of our stars are disk giants ( $76 \%$ of the sample), but we also found a non-negligible amount of stars that belong to the thick disk population, with high space velocity, and low $[\mathrm{Fe} / \mathrm{H}](22 \%)$, according to the above criteria. We also found a small number of halo stars (six objects), which represents $2 \%$ of the sample.

\subsection{Observations and data reduction}

The observational material used in this paper are high resolution, high quality ( $S / N \geq 200$ at $569 \mathrm{~nm}$, typically) optical spectra collected for the PTPS since 2004 with the HET (Ramsey et al. 1998) located in McDonald Observatory. The telescope was equipped with the High Resolution Spectrograph (HRS) that is fed with a 2 arcsec fiber. The HRS was used in the $R=60000$ mode (Tull 1998). Observations were performed using the queue scheduled mode (Shetrone et al. 2007). The spectra consisted of 46 echelle orders recorded on the "blue" CCD chip (407.6-592 nm) and 24 orders on the "red" one (602-783.8 nm).

Since the main goal of PTPS is to obtain high precision radial velocities, the observations were performed in two modes: (i) with the gas $\left(I_{2}\right)$ cell inserted in the optical path and (ii) without the gas cell (GC1 and GC0, respectively). For every target, at least one high $\mathrm{S} / \mathrm{N}(\geq 300)$ GC0 spectrum and a series of $\mathrm{GC} 1$ exposures were obtained. The GC1 exposures contain the $I_{2}$ absorption spectrum imprinted in the first 17 orders of the "blue" spectra and are, therefore, of little use for further spectroscopic studies. For the purpose of this paper, we used all available GC0 exposures and all "red" CCD spectra where the $I_{2}$ lines are not present. Multiple "red" spectra per target allowed for good uncertainty estimates in the case of spectral lines redwards $\sim 660 \mathrm{~nm}$. For spectral lines present in the "blue" CCD, typically only one measurement was possible.

The basic data reduction was performed using standard IRAF $^{3}$ tasks and scripts developed for PTPS. The wavelength scale was corrected to the barycenter of the Solar System.

Unfortunately, the HET/HRS flat-field spectra were sometimes contaminated with an emission spectral features near the ${ }^{7} \mathrm{Li} \lambda 670.8 \mathrm{~nm}$ and $\mathrm{Na}$ D1 and D2 lines. Those features may mimic the stellar absorption lines, depending on the actual RV of a star, and influence the abundance analysis, which leads to artificially increased values. As this features occurred in the flatfields in a more or less random manner, all existing flat-field spectra had to be checked to avoid contamination.

After rejecting all contaminated flat-field spectra, we used the uncontaminated ones from the same night if available.

3 IRAF is distributed by the National Optical Astronomy Observatories, which are operated by the Association of Universities for Research in Astronomy, Inc., under cooperative agreement with the National Science Foundation. 
Otherwise, we used data from the closest night. To avoid introducing additional noise in the reduced spectra in those cases, where only flat-field frames that are distant in time to the original data were available, we cross-correlated them first with each other to determine possible shifts on the CCD matrix.

Another problem we encountered in the "red" CCD reduction process was severe fringing for the data obtained before the new camera was installed in 2006. Fringe effects can be usually compensated by a proper flat-field correction. However, when the emission structure in the flat-field frames and the fringing appeared together, the replaced flat-field frames were not sufficient to properly remove the fringe pattern. In such cases, defective frames were rejected and excluded from further analysis.

To ensure that the instrumental effects described above were properly removed during data reduction, spectra acquired with several other instruments were used to test the reliability of our results (Sect. 3.2). To that end, seven high-resolution spectra (for five stars), which were obtained using the Spanish service time mode at the Instituto de Astrofisica de Canarias at La Palma observatory (Canary Islands, Spain), were obtained the night of the 24 of August 2012 with FIES (Frandsen \& Lindberg 1999) at the Nordic Optical Telescope $(2.56 \mathrm{~m})$. The FIES spectra cover the wavelength range from 364 to $736 \mathrm{~nm}$ with a resolution of $R \sim 67000$. They were reduced using the advanced option of the automatic data reduction tool FIEStool ${ }^{4}$.

We also used 15 spectra of another six targets from our ongoing program using the High Accuracy Radial velocity Planet Searcher-North (HARPS-N) spectrograph at the $3.58 \mathrm{~m}$ Telescopio Nazionale Galileo (TNG; Cosentino et al. 2012). The HARPS-N, a near twin of the HARPS instrument in operation on the ESO $3.6 \mathrm{~m}$ telescope at La Silla (Chile), covers the wavelength range from $380 \mathrm{~nm}$ to $690 \mathrm{~nm}$ with a resolving power of $R \sim 115000$. The standard, automatic reduction pipeline was used.

One additional spectrum obtained at the MERCATOR telescope $(1.2 \mathrm{~m})$ with the HERMES spectrograph (Raskin et al. 2011; Maldonado et al. 2013) was also used for our tests. The HERMES spectra have a resolution of $R \sim 85000$, cover the spectral range $\lambda 380-900 \mathrm{~nm}$, and were automatically reduced using the data reduction pipeline of the instrument ${ }^{5}$.

All spectra used for independent verification of our HET/HRS data had $\mathrm{S} / \mathrm{N}$ values between 50 and 150 around the lines of interest.

We also compared the lithium abundance obtained for Arcturus based on the analysis of PTPS spectra with data available from the literature (see Sect. 3.7.1).

\section{Spectral analysis}

\subsection{Abundances determination}

The spectral analysis was performed with the Spectroscopy Made Easy package (SME, Valenti \& Piskunov 1996). This tool allows us to fit a synthetic spectrum for a star of given spectral type and RV to the observed one. As output, one obtains abundances of the chosen elements and refined stellar parameters. The SME the assumes local thermodynamical equilibrium and plane parallel geometry but ignores the effects of stellar magnetic fields and mass loss. For constructing synthetic spectra, we

\footnotetext{
4 See http://www.not.iac.es/instruments/fies/fiestool/ FIEStool .html for details.

5 See http://www.mercator.iac.es/instruments/hermes/for details.
}

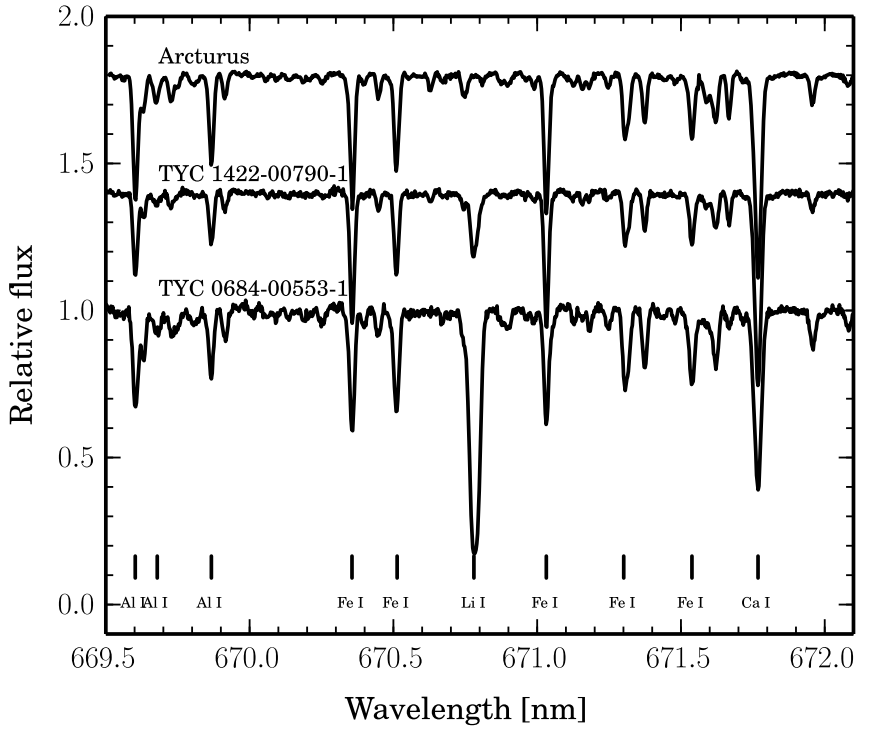

Fig. 2. Spectral region used for the $\mathrm{Li}$ abundance determination using the SME package. The HET spectra of three representative stars are shown.

used 1D-LTE plane-parallel atmosphere models from ATLAS9 by Kurucz (1993).

The SME requires a list of atomic data for lines to be analyzed in the format provided by the Vienna Atomic Line Database (VALD, Kupka et al. 1999) as an input parameter. For every spectral range to be studied, we extracted a starting list of lines representative of an "average" giant star in our sample from VALD, which is a star with $T_{\text {eff }}=4700 \mathrm{~K}, \log g=2.8$, and $v_{\text {mic }}=1.4$. That list was later revised and updated, as described in Sects. 3.2-3.4.

Stellar parameters that are required as the input of $\operatorname{SME}\left(T_{\mathrm{eff}}\right.$, $\log g, v_{\text {mic }},[\mathrm{Fe} / \mathrm{H}]$, and RV) were adopted directly from Zieliński et al. (2012) and were kept as fixed in analysis. The $[\mathrm{Fe} / \mathrm{H}]$ was assumed to be the closest proxy for the stellar metallicity $[\mathrm{M} / \mathrm{H}]$. When solving for individual abundances, the SME uses $[\mathrm{M} / \mathrm{H}]$ as a scaling factor to interpolate between models of atmospheres.

There are three major factors that influence spectral line broadening without changing its depth: instrumental profile, macroturbulence velocity $\zeta$, and rotation velocity. We defined the instrumental profile as a Gaussian function parametrized by spectral resolution. In case of PTPS spectra, this is 60000 . It is assumed, that broadening profiles for macroturbulence and rotation velocity are Gaussian in shape in SME, but the input value for $\zeta$ is actually the radial tangential macroturbulence parameter, rescaled to the Gaussian macro turbulence by using a factor of $\sqrt{2}$. For giants, rotation velocity contributes less to line broadening than macroturbulence. Hence, we adopted $v \sin i=0$ and solved only for macro turbulence. We present our rotation velocity determination method in Sect. 3.6.

\subsection{Lithium abundance}

The Li abundance analysis was performed using only the order of the "red" HET/HRS spectra, which contains the spectral range from $\lambda 670.3$ to $671.1 \mathrm{~nm}$ (see Fig. 2). There are no $I_{2}$ lines present in that range, and we used all available GC1 spectra. Their actual number depends on the star status in the PTPS observing program and can range from 2 to about 100 per star. 
M. Adamów et al.: The Penn State - Toruń Centre for Astronomy Planet Search stars. II.

Table 1. $A(\mathrm{Li})$ determinations based on spectra obtained with different instruments.

\begin{tabular}{llccccc}
\hline \hline Star & & $A(\mathrm{Li})_{\text {HET }}$ & $\sigma_{s, \mathrm{HET}}$ & $A(\mathrm{Li})$ & $\sigma_{\mathrm{s}}$ & Instrument \\
\hline TYC 0870-00114-1 & HIP 57428 & $<0.63$ & & 0.89 & & HERMES \\
\hline TYC 0435-03332-1 & BD+02 3497 & 1.44 & 0.01 & 1.42 & 0.01 & FIES \\
TYC 1422-00790-1 & BD+20 2457 & 0.32 & 0.01 & 0.29 & 0.02 & FIES \\
TYC 3226-02285-1 & HIP 111944 & 0.73 & 0.01 & 0.74 & - & FIES \\
TYC 3304-00101-1 & HD 17092 & $<-0.10$ & & $<-0.26$ & & FIES \\
TYC 4006-00980-1 & HD 240210 & $<-0.17$ & & $<-0.80$ & & FIES \\
\hline TYC 0684-00553-1 & & 3.06 & 0.02 & 3.16 & 0.01 & HARPS-N \\
TYC 3226-01219-1 & HD 216016 & 1.10 & 0.01 & 1.15 & 0.01 & HARPS-N \\
TYC 3304-00090-1 & BD+48 740 & 1.90 & 0.01 & 1.94 & 0.01 & HARPS-N \\
TYC 3917-01107-1 & HD 238914 & 1.83 & 0.01 & 1.85 & - & HARPS-N \\
TYC 3993-01850-1 & & 1.75 & 0.07 & 1.80 & 0.02 & HARPS-N \\
TYC 4421-01779-1 & HD 160723 & 1.04 & 0.03 & 1.07 & - & HARPS-N \\
\hline
\end{tabular}

Notes. All abundance values are given in dex.

We analyzed 4750 spectra in total with 14 spectra per star on average.

With SME, we fitted the ${ }^{7} \mathrm{Li}$ line at $\lambda 670.8 \mathrm{~nm}$ with several lines of $\mathrm{C}, \mathrm{N}, \mathrm{V}, \mathrm{Sm}$, and $\mathrm{Fe}$ that are present in that spectral region and the macroturbulence velocity. All initial abundance values are solar, except for $\mathrm{C}$ and $\mathrm{N}$, which abundances we expect to be processed by first dredge up. We therefore adopted abundances typical for a red giant: $\mathrm{C} / \mathrm{H}=-4.01$ and $\mathrm{N} / \mathrm{H}=-4.07$ (Iben 2013).

We replaced the initial VALD list of lines with that from Carlberg et al. (2012) but we expanded it with two Fe lines at $\lambda 670.36$ and $671.03 \mathrm{~nm}$, for which we used line data from VALD $(\log g f=-3.16,-4.88$, respectively). For vast majority of giants analyzed here, $\mathrm{Li}$ lines, as well as nearby $\mathrm{CN}$ and elemental lines are weak, blended structures, therefore the two Fe lines were included to control RV shift (we allowed SME for small adjustments of RV) and line broadening.

To check if the instrumental effects discussed in Sect. 2 were properly removed, we analyzed spectra of 12 stars from our sample obtained with other telescopes and instrumental configurations. Our Li abundances obtained from HET spectra and those obtained from HERMES, FIES, and HARPS-N spectra are presented in Table 1, where Col. (1) lists the target TYCHO name and Col. (2) an alternate identification; Cols. (3) and (4) provide lithium abundances derived using HET spectra and their uncertainties $\left(\sigma_{\mathrm{s}}\right.$, which describe spectra quality only, whenever multiple spectra per target were available, see also Sect. 3.5). Columns (5) and (6) list the lithium abundances and uncertainties obtained from instruments listed in Col. (7). Of all stars presented in Table 1, only one, TYC 3226-02285-1, was observed during nights with all flat-field frames not containing emission lines. For the rest of stars, the number of observing nights for which all flats were contaminated by the emission lines varied from 1 for TYC 3226-01219-1 to 18 for TYC 3917-01107-1. The Li abundances determined from spectra obtained with different instruments are very similar, which are typically within estimated uncertainties of individual determination, to those obtained from HET/HRS spectra. These comparisons give us confidence on the validity of the procedure for the proper removal of instrumental effects in the HET data and, therefore, on the reliability of the abundances derived from HET/HRS data.

Another important issue to consider is that the lithium line at $\lambda 670.8 \mathrm{~nm}$, which is the base of our analysis, may be subject to several non-LTE processes, such as photon loss, ultraviolet overionization, and bound-bound pumping (Carlsson et al. 1994, and references therein). All these processes are ignored in SME analysis and thus need to be taken into account separately. We applied the non-LTE corrections provided by Lind et al. (2009) to our LTE lithium abundances for a star of given $T_{\text {eff }}, \log g, \quad v_{\text {mic }}$ and $[\mathrm{Fe} / \mathrm{H}]$. For three stars, $v_{\text {mic }}$ fell outside the range of Lind et al. (2009) grids, and we applied a nonLTE correction for the maximum available value of $2 \mathrm{~km} \mathrm{~s}^{-1}$. For 63 objects, our LTE Li abundances were below the minimum value, for which curves of growth are defined in Lind et al. (2009). For those stars, we adopted non-LTE correction for the lowest LTE value provided. For most of the stars in our sample, the non-LTE corrections are on the order of 0.2 dex.

\subsection{Oxygen abundance}

Oxygen abundances, $[\mathrm{O} / \mathrm{H}]$ were obtained from the analysis of the $\lambda 777.2,777.4$, and $777.5 \mathrm{~nm}$ triplet and, separately, from three lines at $\lambda 557.7,630.0$, and $636.3 \mathrm{~nm}$.

Atomic data for each line of the triplet were adopted directly from VALD $(\log g f=0.369,0.223,0.001$, respectively). Those spectral features appear in two of the HET/HRS "red" spectra echelle orders with slightly different signal-to-noise ratio. We separately extracted the spectral lines from both orders, doubling the number of input files introduced into SME for the analysis. The spectral range of the oxygen triplet is beyond any possible contamination from the $I_{2}$ absorption, so we used all available GC1 spectra for every star (between two and 100 spectra, 14 on average). The analysis was performed separately for each line of the triplet. Since the oxygen triplet is strongly affected by nonLTE effects, we applied the non-LTE corrections by Ramírez et al. (2007) to the abundances derived using SME. For 59 stars with $T_{\text {eff }}<4400 \mathrm{~K}, \log g<2$ and/or $[\mathrm{Fe} / \mathrm{H}]>0.4$ stellar parameters fell out of the non-LTE grids and extrapolated non-LTE values were used.

For the three oxygen lines at $\lambda 557.7,630.0$, and $636.3 \mathrm{~nm}$, $\log g f$ data were updated using line list by Ramírez \& Allende Prieto (2011). Those lines are less affected by nonLTE effects but blended ( $\lambda 557.7 \mathrm{~nm}$ with $\mathrm{Y}, \lambda 630.0$ with $\mathrm{Ni}$ ) and available only in limited number of GC0 spectra. No nonLTE correction was applied to oxygen abundances obtained from those three lines.

Both oxygen abundances agree within uncertainties (see Sect. 3.5 for more detailed discussion on uncertainties estimates). Hence, the final oxygen abundance presented is the average of those two values. 


\subsection{Aluminium, magnesium, titanium, and calcium abundances}

Abundances for all four elements were determined using narrow $0.1-0.22 \mathrm{~nm}$ ranges of spectra, centered on unblended lines. We used $\mathrm{Al}, \mathrm{Mg}$, Ti, and Ca lines in the range $\lambda 520-660 \mathrm{~nm}$ selected by Ramírez \& Allende Prieto (2011). We also updated the initial VALD line list using $\log g f$ from that paper. Lines of $\mathrm{Mg}, \mathrm{Ca}$ and Ti are present in spectral regions strongly affected by $I_{2}$ lines. Hence, we used only GC0 spectra for SME analysis. Lines of $\mathrm{Al}$ are not affected by iodine spectra. Therefore, both GC0 and GC1 spectra were in use.

We did not apply non-LTE corrections to the obtained abundances. Non-LTE corrections of $\mathrm{Al}, \mathrm{Mg}, \mathrm{Ti}$ and $\mathrm{Ca}$ are usually calculated for metal-poor stars (halo stars and stars in globular clusters) or are for far infrared lines (beyond $\lambda 1000 \mathrm{~nm}$ ). Most of our stars fall out of the range of stellar parameters provided in the available grids (see e.g. Spite et al. 2012 for Ca). Non-LTE corrections for solar-metallicity giants are sorely needed (Luck \& Heiter 2007).

\subsection{Abundances uncertainties}

There are several sources of uncertainties in abundance analysis. One of them are uncertainties connected with the quality of spectra (variable signal-to-noise ratio, continuum fitting, and changes in the instrumental profile). The availability of multiple spectra for every single star represents an advantage for the uncertainty calculation. Next are uncertainties of line-to-line variations whenever analysis includes more than one line per element. Uncertainties introduced by stellar parameters adopted in analysis should also be taken into account. In the case of $\mathrm{Li}$ and $\mathrm{O}$, application of non-LTE corrections also contributes to the final uncertainties.

The contribution to the uncertainties of spectra quality and line variations, $\sigma_{\mathrm{s}}$ were estimated from the standard deviation obtained from multi-epoch observations and scatter in abundances due to multiple lines analysis for a particular element. The average uncertainties for stellar parameters adopted from Zieliński et al. (2012) are: $\Delta T_{\text {eff }}=40 \mathrm{~K}, \Delta \log g=$ $0.15, \Delta[\mathrm{Fe} / \mathrm{H}]=0.07$, and $\Delta v_{\text {mic }}=0.24$. We estimated their contributions to the final uncertainties, $\left(\sigma_{\mathrm{T}}, \sigma_{\mathrm{g}}, \sigma_{\mathrm{Fe}}\right.$ and $\sigma_{\xi}$, respectively) by varying the input parameters by those values. The results are presented in Table 2 . The total uncertainty is a quadratic sum of all sources of uncertainties listed. As the number of individual determinations was typically small $(<30)$, the standard deviation is multiplied by the t-Student ${ }^{6}$ coefficient for confidence level of $68 \%$ (i.e. $1 \sigma)-\alpha=0.32$.

For Li abundances, the uncertainty was calculated from multiple LTE determinations. Uncertainty estimates for the non-LTE corrections for $\mathrm{Li}$ were not available. The final $\mathrm{Li}$ uncertainties are therefore those resulting from LTE analysis.

For oxygen triplets, multiple abundance determinations were available. The final uncertainty was then calculated by averaging the uncertainties obtained for each single line of the triplet. The procedure to calculate the non-LTE correction for O did not take into account the uncertainty in the abundance determination as an input parameter, but it returned an line-to-line uncertainty. Thus, for oxygen triplet abundances, the $\sigma_{\mathrm{s}}$ is a quadratic sum of the uncertainties resulting from the instrumental effects and the uncertainties from non-LTE corrections.

\footnotetext{
6 Small-number t-Student statistics by William Sealy Gosset ("Student") was first described in Student (1908).
}

Table 2. Uncertainties in abundance analysis propagated from stellar parameters errors.

\begin{tabular}{c|cccc}
\hline \hline & $\sigma_{\mathrm{T}}$ & $\sigma_{\mathrm{g}}$ & $\sigma_{\mathrm{Fe}}$ & $\sigma_{\xi}$ \\
\hline $\mathrm{Li}$ & 0.06 & 0.14 & 0.13 & 0.03 \\
$\mathrm{O}_{t}$ & 0.08 & 0.09 & 0.10 & 0.10 \\
$\mathrm{O}$ & 0.01 & 0.04 & 0.02 & 0.01 \\
$\mathrm{Mg}$ & 0.05 & 0.02 & 0.08 & 0.06 \\
$\mathrm{Al}$ & 0.03 & 0.01 & 0.07 & 0.04 \\
$\mathrm{Ca}$ & 0.04 & 0.07 & 0.06 & 0.10 \\
$\mathrm{Ti}$ & 0.07 & 0.02 & 0.03 & 0.10 \\
\hline
\end{tabular}

For the three oxygen lines at $\lambda 557.7,630.0$ and $636.3 \mathrm{~nm}$, the abundance uncertainty was calculated from the scatter in three determinations.

One should mind the differences for oxygen: the triplet lines are more sensitive to stellar parameters, while the line-to-line uncertainties for the other three LTE lines and poor statistic (high t-Student coefficients) strongly contributed to total uncertainty. In both cases, the average uncertainty for oxygen abundance is 0.2 dex.

\subsection{Rotational velocities}

In the case of giants, rotational velocities are expected to be smaller than macro turbulence velocities and, hence, contribute less to the total line broadening. We have, however, estimated them within our spectral analysis, as rotation could be related to the lithium overabundances; the nature of which we are trying to understand. We obtained the rotational velocities using two independent methods: by measuring the line width through cross correlation as described in Nowak et al. (2013) and by SME spectral modeling. In both methods, the macroturbulence velocity $\zeta_{\text {HM }}$ was adopted from the Hekker \& Meléndez (2007) calibrations for every star, which is an effective temperature and surface gravity combination. We assumed, that rotational velocity can be expressed as:

$v \sin i=\sqrt{\beta^{2}-C \zeta_{\mathrm{HM}}^{2}}$,

where $\beta$ stands for the total line broadening (assumed to be Gaussian) and $C$ is a factor scaling the radial-tangential $\zeta_{\mathrm{HM}}$ values based on work by Gray and collaborators with Doppler shift distributions for macroturbulence velocity (Massarotti et al. 2008; Fekel 1997). If the respective $\zeta_{\mathrm{HM}}$ from Hekker \& Meléndez (2007) was higher than the total line broadening, we followed the widely accepted procedure and adopted $\zeta_{\mathrm{HM}}$ for lower luminosity class.

The cross-correlation functions (CCFs) were computed by cross-correlating the stellar spectra (GC0) with a numerical mask consisting of 1 and 0 value points with the non-zero points corresponding to the positions of the stellar absorption lines at zero velocity. The mask was constructed from a synthetic K2 star spectrum of Kurucz (1993) ATLAS9 and contained about 300 lines. The CCFs were computed in all the 17 HRS orders, which cover $\lambda 504-592 \mathrm{~nm}$ spectral range and are used for RV measurements over a wide $\pm 30 \mathrm{~km} \mathrm{~s}^{-1}$ range after correcting the spectra for their absolute radial velocities and shifting them to the Solar System barycenter (Stumpff 1980). Finally, the $17 \mathrm{CCFs}$ were added together in the wavelength scale to form the integrated CCF. To determine $v \sin i$, we followed the procedure from Carlberg et al. (2011) but assumed $C=0.5$ and subtracted the instrumental profile of $5 \mathrm{~km} \mathrm{~s}^{-1}$. 
Table 3. Comparison of $\alpha$-elements abundances for Arcturus.

\begin{tabular}{c|ccc|c|c}
\hline \hline & \multicolumn{3}{|c|}{ This work } & Ramírez \& Allende Prieto (2011) & Smith et al. (2013) \\
$\mathrm{x}$ & $A(\mathrm{x})_{\odot}$ & $A(\mathrm{x})$ & {$[\mathrm{x} / \mathrm{H}]$} & {$[\mathrm{x} / \mathrm{H}]$} & $8.64 \pm 0.04$ \\
$\mathrm{O}$ & 8.62 & $8.29 \pm 0.14$ & $-0.28 \pm 0.14$ & $-0.02 \pm 0.03$ & $7.15 \pm 0.08$ \\
$\mathrm{Mg}$ & 7.49 & $7.14 \pm 0.14$ & $-0.35 \pm 0.14$ & $-0.15 \pm 0.03$ & $6.16 \pm 0.01$ \\
$\mathrm{Al}$ & 6.33 & $6.14 \pm 0.10$ & $-0.19 \pm 0.10$ & $-0.18 \pm 0.03$ & $5.84 \pm 0.07$ \\
$\mathrm{Ca}$ & 6.27 & $5.73 \pm 0.14$ & $-0.54 \pm 0.14$ & $-0.41 \pm 0.04$ & $4.59 \pm 0.07$ \\
$\mathrm{Ti}$ & 4.86 & $4.55 \pm 0.24$ & $-0.32 \pm 0.14$ & $-0.25 \pm 0.05$ & $6.98 \pm 0.04$ \\
$\mathrm{Fe}$ & 7.41 & $6.89^{a}$ & $-0.52^{a}$ & $-0.52 \pm 0.04$ & \\
\hline
\end{tabular}

Notes. ${ }^{(a)}$ Abundance value adopted from Ramírez \& Allende Prieto (2011).

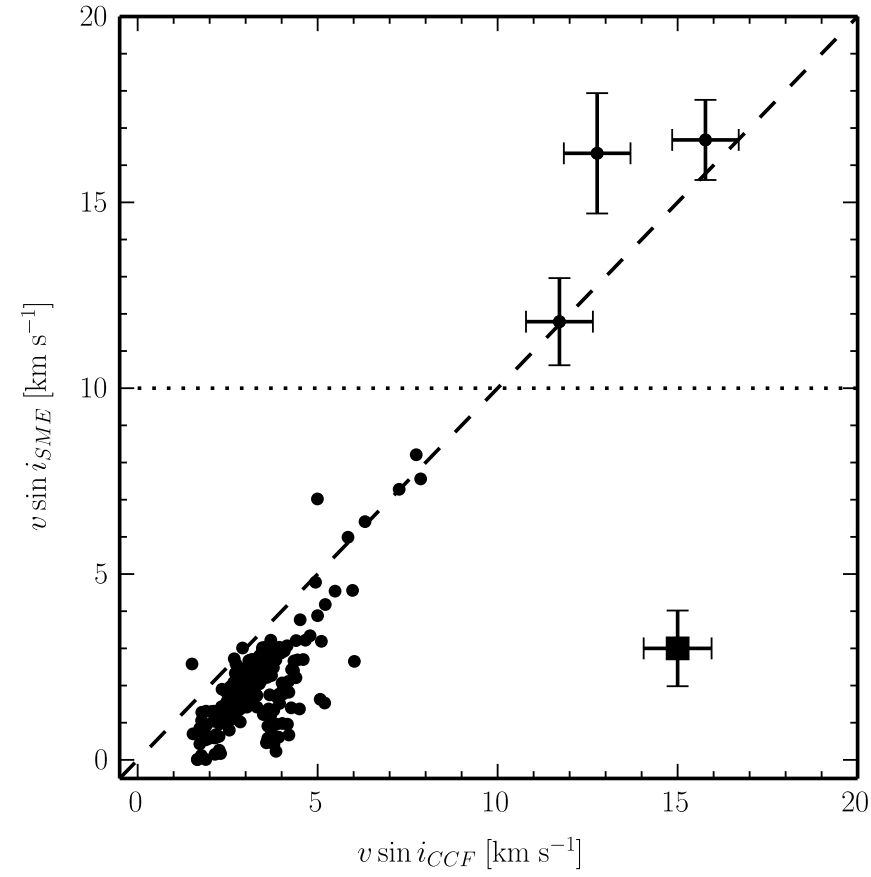

Fig. 3. Stellar rotation velocities obtained using the cross-correlation technique $-v \sin i_{\mathrm{CCF}}$ and those obtained from SME synthetic spectrum fitting $-v \sin i_{\mathrm{SME}}$. The dashed line marks a one-to-one relation. The dotted one separates the fast and slow rotators. In the lower right corner, an average uncertainty in $v \sin i$ determination (for slow rotating stars) for both methods is presented.

In SME analysis, we measured line broadening by assuming that it stems from macro turbulence only. This allowed us to obtain a parameter which should be interpreted as an upper limit for the radial-tangential macro turbulence $\beta_{\zeta}$. Assuming a theoretical macroturbulence velocity $\zeta_{\mathrm{HM}}$ from the Hekker $\&$ Meléndez (2007) calibrations for every star, we modyfied Eq. (1) and estimated the rotation velocity $v \sin i$ as:

$v \sin i=\sqrt{C\left(\beta_{\zeta}^{2}-\zeta_{\mathrm{HM}^{2}}\right)}$.

Uncertainties in the rotational velocities were calculated by taking dispersions in the total broadening and $\zeta_{\mathrm{HM}}$ fitting into account.

Figure 3 shows that the rotational velocities obtained with the two methods are generally in good agreement. Three rapid rotators, which are defined usually as stars with $v \sin i>$ $10 \mathrm{~km} \mathrm{~s}^{-1}$ (Fekel 1997) are confirmed. These are: TYC 393001790-1, as identified already by Massarotti et al. (2008), and
TYC 3676-02387-1 and TYC 3318-00020-1, which are both detected by Nowak (2012).

For stars with $v \sin i<5 \mathrm{~km} \mathrm{~s}^{-1}$, the SME analysis revealed rotational velocities systematically lower by $\sim 1 \mathrm{~km} \mathrm{~s}^{-1}$ as compared to those obtained with CCFs, which is probably due to different methodology applied to their determination. A small group of stars, slow rotators, for which $v \sin i_{\mathrm{CCF}}-v \sin i_{\mathrm{SME}} \gtrsim$ $2 \mathrm{~km} \mathrm{~s}^{-1}$ is also present in Fig. 3. These are objects, for which $\beta<\zeta_{\mathrm{HM}}$ and realistic $v \sin i_{\mathrm{CCF}}$ determination required a change in luminosity class to obtain $\zeta_{\mathrm{HM}}$ from Hekker \& Meléndez (2007) calibrations, while the correct and hence higher $\zeta_{\mathrm{HM}}$ could be applied to calculate $v \sin i_{\mathrm{SME}}$.

\subsection{Consistency check}

\subsubsection{Arcturus abundances}

To test the aforementioned methodology, we applied it to Arcturus. Three very good quality $(S / N \sim 300)$ HET/HRS spectra in standard PTPS configuration were obtained on 16 Jan., 27 Jan. and 20 Feb. 2012. Our Li abundance, $A(\mathrm{Li})_{\text {LTE }}<-0.74$, agrees very well with the determinations of Carlberg et al. (2012) with $A(\mathrm{Li})<-0.73$, Reddy \& Lambert (2005) with $A(\mathrm{Li})<$ -0.6 and Brown et al. (1989) with $A(\mathrm{Li})<-0.8$, despite that all flats were contaminated with emission lines. In Table 3 we present $\alpha$-elements abundances for Arcturus obtained here and compare them to recent results of several other authors. We also present the adopted solar values used in calculation of $[\mathrm{X} / \mathrm{H}]$ through this paper in Table 3. For Al, Ca, and Ti, our abundances are in very good agreement with those obtained by Ramírez \& Allende Prieto (2011) and Smith et al. (2013). In case of Mg, we practically obtained identical value as Smith et al. (2013). Our oxygen abundance is lower by 0.3 dex than those presented by Ramírez \& Allende Prieto (2011) and Smith et al. (2013). We note, however, that the oxygen abundance for three LTE lines is $[\mathrm{O} / \mathrm{H}]=-0.24 \pm 0.09$ and the oxygen abundance discrepancy is not a result of extrapolated non-NLTE correction for the triplet lines.

Arcturus is a slow-rotating star. Our rotational velocity of $v \sin i_{\mathrm{SME}}=2.7 \pm 0.6 \mathrm{~km} \mathrm{~s}^{-1}$ agrees very well with those of Carlberg et al. (2012) at $2 \pm 1.5 \mathrm{~km} \mathrm{~s}^{-1}$ and Carney et al. (2008) at $2.4 \pm 1 \mathrm{~km} \mathrm{~s}^{-1}$.

\subsection{2. $\alpha$-elements abundances vs. metallicity}

Abundances of $\mathrm{O}, \mathrm{Mg}, \mathrm{Al}, \mathrm{Ca}$, and $\mathrm{Ti}$ as a function of $[\mathrm{Fe} / \mathrm{H}]$ are shown in Fig. 4, where we also present trends of $[\mathrm{X} / \mathrm{H}]$ with $[\mathrm{Fe} / \mathrm{H}]$ as obtained by Luck \& Heiter (2007) and fits to these relations. Many of stars with $[\mathrm{Fe} / \mathrm{H}]<-0.6$ are a thick disk or halo population objects that seem to form a different relation. 
Table 4. Coefficients for linear fits of $[\mathrm{Fe} / \mathrm{H}]$ vs. $[\mathrm{X} / \mathrm{H}]$ trend. $([\mathrm{X} / \mathrm{H}]=$ slope $\times[\mathrm{Fe} / \mathrm{H}]+$ intercept.)

\begin{tabular}{c|cc}
\hline \hline $\mathrm{X}$ & Slope & Intercept \\
\hline $\mathrm{O}$ & $0.705 \pm 0.103$ & $0.149 \pm 0.024$ \\
$\mathrm{Mg}$ & $0.574 \pm 0.029$ & $0.015 \pm 0.007$ \\
$\mathrm{Al}$ & $0.771 \pm 0.064$ & $0.092 \pm 0.015$ \\
$\mathrm{Ca}$ & $0.915 \pm 0.030$ & $-0.031 \pm 0.007$ \\
$\mathrm{Ti}$ & $0.818 \pm 0.036$ & $0.035 \pm 0.009$ \\
\hline
\end{tabular}
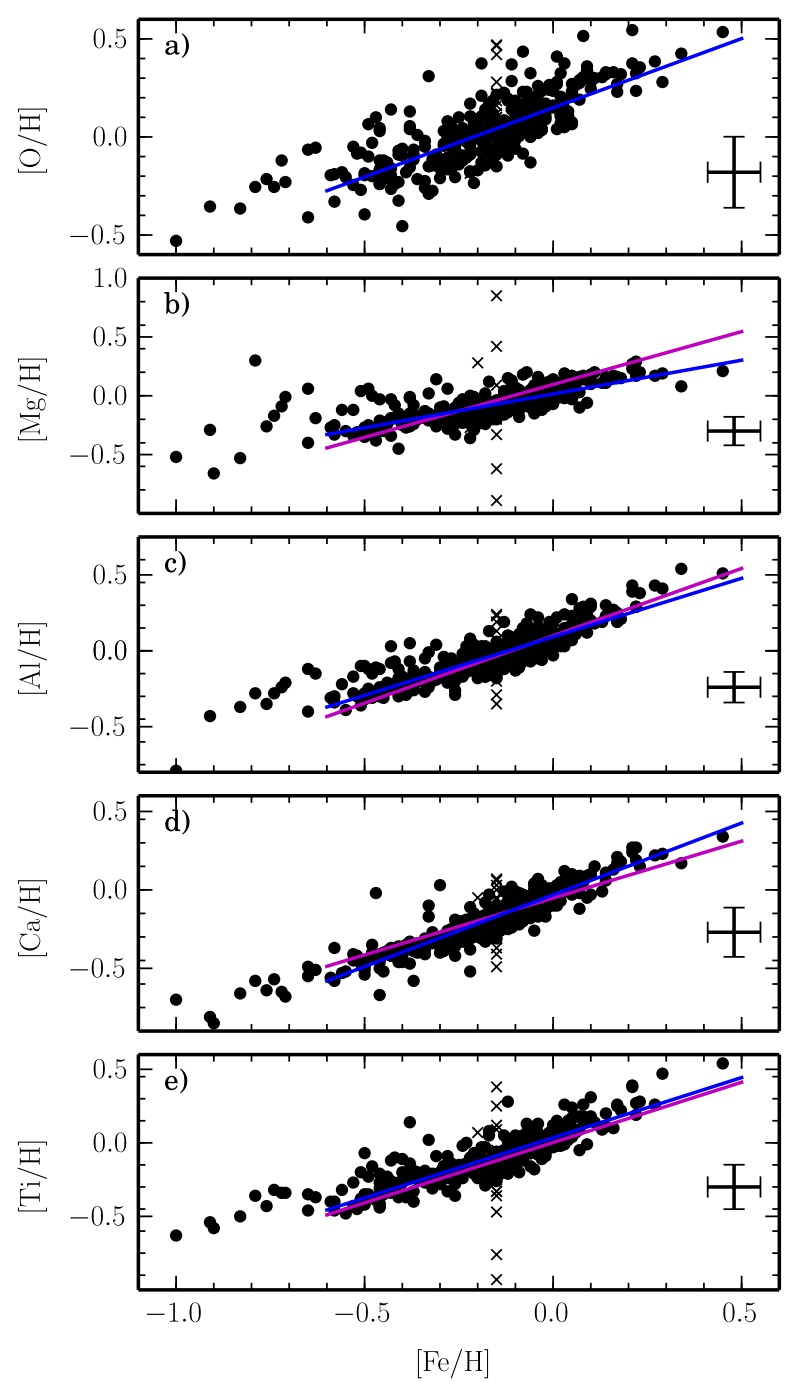

Fig. 4. Abundances for the complete sample of giants (crosses denote stars with uncertain atmospheric parameters). The error bars in the lower right corners are the mean uncertainties for $[\mathrm{Fe} / \mathrm{H}]$, as adopted from Zieliński et al. (2012) and for abundances of a given element obtained in this work. Blue lines represent linear regression fits with coefficients presented in Table 4, while magenta lines are fits of Luck \& Heiter (2007).

Therefore, we fitted only stars with $[\mathrm{Fe} / \mathrm{H}]>-0.6$ to compare our results with Luck \& Heiter (2007). Coefficients for linear fits to our data for each element are presented in Table 4. General relations between elemental abundances and $[\mathrm{Fe} / \mathrm{H}]$ agree with those of Luck \& Heiter (2007), and we are confident that our $\alpha$-elements abundances are correct.

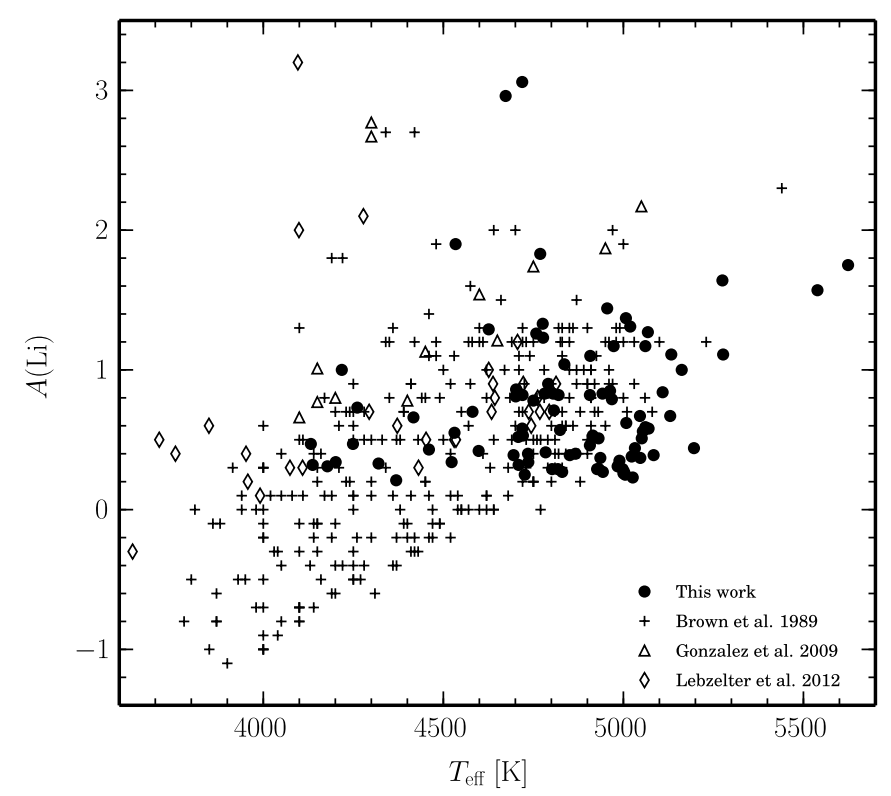

Fig. 5. Comparison between the lithium abundances and stellar effective temperatures obtained in this work with those obtained by Lebzelter et al. (2012), Gonzalez et al. (2009), and Brown et al. (1989). For consistency, our $A(\mathrm{Li})_{\text {LTE }}$ determinations are presented for 92 stars (5 dwarfs, 2 subgiants and 85 giants) with detected Li.

\section{Results - general}

Our abundance determinations for $\mathrm{Li}, \mathrm{O}, \mathrm{Mg}, \mathrm{Al}, \mathrm{Ca}$, and $\mathrm{Ti}$ as well as rotational velocities with their derived uncertainties for all investigated objects are presented in Table 5. For each of the 348 stars analyzed, Col. (1) gives the target name according to the TYCHO catalog; Cols. (2)-(5) list the average rotation velocities obtained with the two methods and their uncertainties. In Col. (6), we give the lithium abundance (or its upper limit) based on multi-epoch observations; Col. (7) contains the lithium abundance after non-LTE correction. Column (8) provides the uncertainty obtained for $A(\mathrm{Li})$. Columns (9) to (18) list the $\mathrm{O}, \mathrm{Mg}, \mathrm{Al}, \mathrm{Ca}$, Ti abundances and their estimated uncertainties, and Col. (19) contains information on the stellar subsystem to which each given star belongs according to Ibukiyama \& Arimoto (2002) criteria. The last Col. (20) provides additional information about particular object. Although the table includes the results for five dwarf stars identified by Zieliński et al. (2012), they have been excluded from further analysis.

Regarding the $\mathrm{Li}$ abundance, which is the main focus of this paper, we identified 92 stars with detectable Li lines in our sample. Most of $A(\mathrm{Li})$ abundances are based on multi-epoch sets of spectra, and each determination was found to be consistent within an estimated $\sigma A(\mathrm{Li})$ level. Among the 92 detections, we identified 12 giants with large $A(\mathrm{Li})$ abundances and two with lithium abundances close to the meteoritic value. For 271 stars with $A(\mathrm{Li})<0$ or $A(\mathrm{Li}) \lesssim \sigma A(\mathrm{Li})$, we provide only upper limits defined as the maximum value of $A(\mathrm{Li})$ for a set of spectra for a given star.

A comparison between the lithium abundances obtained in this paper and those available from the literature is presented in Fig. 5. The results of extended spectral analysis of Brown et al. (1989) based on high resolution spectra for 644 field stars, and data from more recent studies by Gonzalez et al. (2009) and Lebzelter et al. (2012), which are focused on stars in the Galactic Bulge, are plotted together with our results. All 


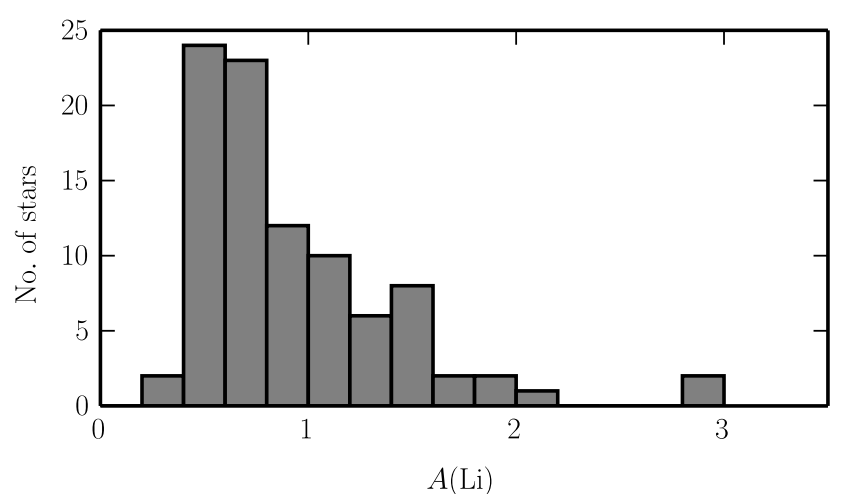

Fig. 6. Histogram of $A(\mathrm{Li})$ for 92 stars with Li detections. We note a jump in the number of stars at $A(\mathrm{Li})>1.4$.

available Li abundances, including those presented here, show an identical tendency regarding lithium abundances and $T_{\text {eff }}$; cooler stars reach lower Li abundances. Our sample is comparable to that analyzed by Brown et al. (1989): it covers similar range in $\log g$ and $T_{\text {eff }}$ and focuses on field stars. The main difference is the more restricted selection of stars in the PTPS. The sample presented here does not include stars with $T_{\text {eff }} \lesssim 4000$ nor are masses greater than $\sim 3.5 M_{\odot}$, while the Brown et al. (1989) sample does. These limitations are the consequence of the observational strategy which is required for searching planets around evolved stars.

In Fig. 6, we present a distribution of obtained $A(\mathrm{Li})$. In addition to a gradual decay, we observe a sudden increase of stars with $A(\mathrm{Li})>1.4$ and a few super-Li giants.

\subsection{Giants with lithium on the Hertzsprung-Russel diagram}

The location of our stars in the Hertzsprung-Rusell (HR) diagram is shown in Fig. 7, where evolutionary tracks of 1 to $3 M_{\odot}$ stars and solar metallicity from Bertelli et al. (2008) are presented as well.

To investigate the Li abundance of our sample of PTPS giants, we have divided the total sample (323 stars) in three groups: (1) stars with only upper limits or no lithium detections (241 objects, group A, black symbols in Fig. 7); (2) stars with detected $\mathrm{Li}$ and $A(\mathrm{Li})_{\mathrm{NLTE}}<1.4$ (71 objects, group $\mathrm{B}$, blue symbols); and (3) giant stars with $\mathrm{Li}$-abundances $A(\mathrm{Li})_{\mathrm{NLTE}}>1.4$ (11 objects, group $\mathrm{C}$, red symbols).

In addition to the five dwarfs identified by Zieliński et al. (2012) we also excluded another two stars: TYC 3676-02387-1 $\left(A(\mathrm{Li})_{\mathrm{NLTE}}\right.$ of 1.75$)$ and TYC 3993-01850-1 $\left(A(\mathrm{Li})_{\mathrm{NLTE}}=1.81\right)$ from the whole further analysis, despite their high lithium abundances. According to their effective temperatures and $\log g$, these two stars are still in the subgiant phase in that they only recently started the first dredge-up, and thus, their high lithium abundances are most likely leftover MS values.

Two more objects, as identified within PTPS as spectroscopic binaries with unresolved lines (TYC 3667-00550-1, TYC 4421-01996-1) and the sixteen stars for which we only have estimates of the stellar parameters from Zieliński et al. (2012) were excluded from statistical analysis for consistency as well. The later stars are presented in the following figures; however, they are marked as crosses colored according to their A(Li).

Given that lithium depletion is not expected in a star on the RGB before the first dredge-up (FDU) has occurred, it is important to determine the location of the star on the HR diagram with

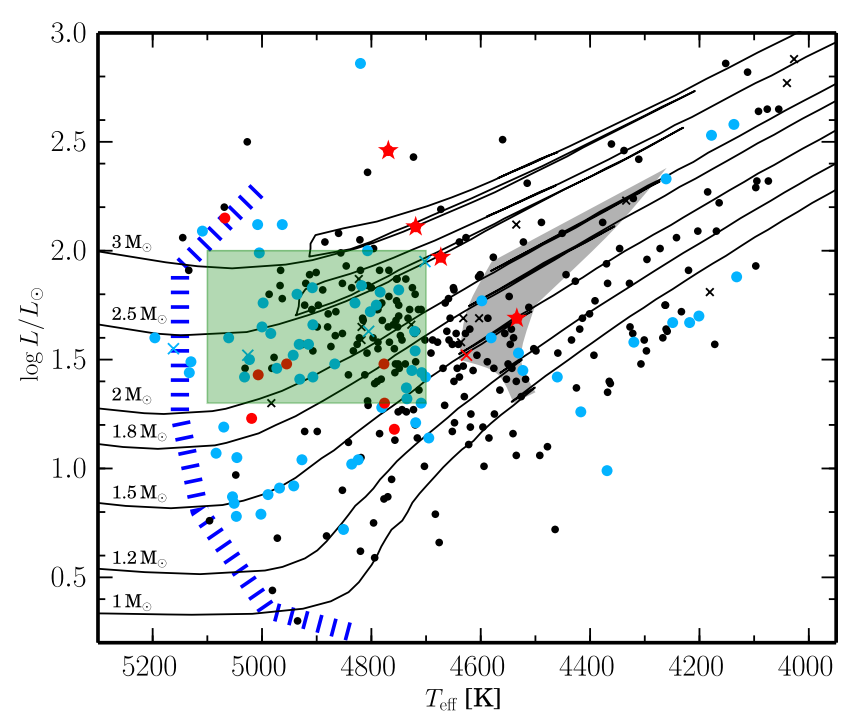

Fig. 7. HR diagram showing the location of all the PTPS stars studied in this paper. Colors of points denote $\mathrm{Li}$ abundance. Red are the most Li-rich objects (the red stars denote the four most Li-rich giants). Stars denoted in blue have moderate $\mathrm{Li}$ level, and black is for Li-poor stars. Crosses denote stars with uncertain atmospheric parameters. The blue stripes denote the beginning of RGB. The gray area is LFB region, and green box denotes RGC. Evolutionary tracks from Bertelli et al. (2008) for $1-3 M_{\odot}$ stars with solar metallicity are plotted as solid lines.

respect to this event. For FGK stars, Li depletion due to the FDU starts at approximately $T_{\text {eff }}=5400 \mathrm{~K}$ and ends at $T_{\text {eff }}=4500 \mathrm{~K}$ (Charbonnel \& Balachandran 2000) when the convective envelope reaches its maximum depth. A higher Li abundance as a remnant from the MS phase could be confused with an overabundance per se if the star did not experience severe FDU dilution yet. This is particularly important for stars in the higher mass end $\approx 1.5-3 M_{\odot}$ and solar metallicity, since Li is not so easily destroyed during the MS lifetime of the star (note, however, the small number of objects we have with solar metallicities). In solar mass stars lithium is strongly depleted during the MS lifetime and they are expected to leave the MS with $A(\mathrm{Li}) \sim 1$.

Points representing the position of the RGB bottom for the presented evolutionary tracks were joined with blue striped line to define the beginning of the RGB for various masses. The light gray area represents the location of the LFB on the first ascent of RGB.

In the green block in Fig. 7 we have marked the location of the RGC region, as defined by $4700 \mathrm{~K}<T_{\text {eff }}<5100 \mathrm{~K}$ and $1.3<\log L / L_{\odot}<2.0$ (see Zieliński et al. 2012). We can see in Fig. 7 that we have a substantial number of stars located in the region that corresponds to the first ascent on the RGB in our sample, and a handful of objects with low $T_{\text {eff }}$ and high luminosities for which we cannot fully exclude the possibility that they are already evolved off the horizontal branch (HB).

Of the 323 giants in our complete sample, 125 (37 $\pm 3 \%)$ fall into an extended RGC region of the HR diagram as defined in Zieliński et al. (2012) and from the sample of 82 giants with a detected Li (group B and C) 35 (43 $\pm 5 \%$ ). Within uncertainties, these fractions agree. In the LFB area of HR diagram, there are $28(9 \pm 2 \%)$ giants from the total sample and five with detected $\mathrm{Li}(6 \pm 3 \%)$. Of 11 giants from group C, only four $(36 \pm 15 \%)$ fall into RGC and one $(9 \pm 9 \%)$ into LFB. Again, within uncertainties, these fractions agree. We noticed (although it is fully discussed later in the paper) that the Li-rich stars are not grouped 

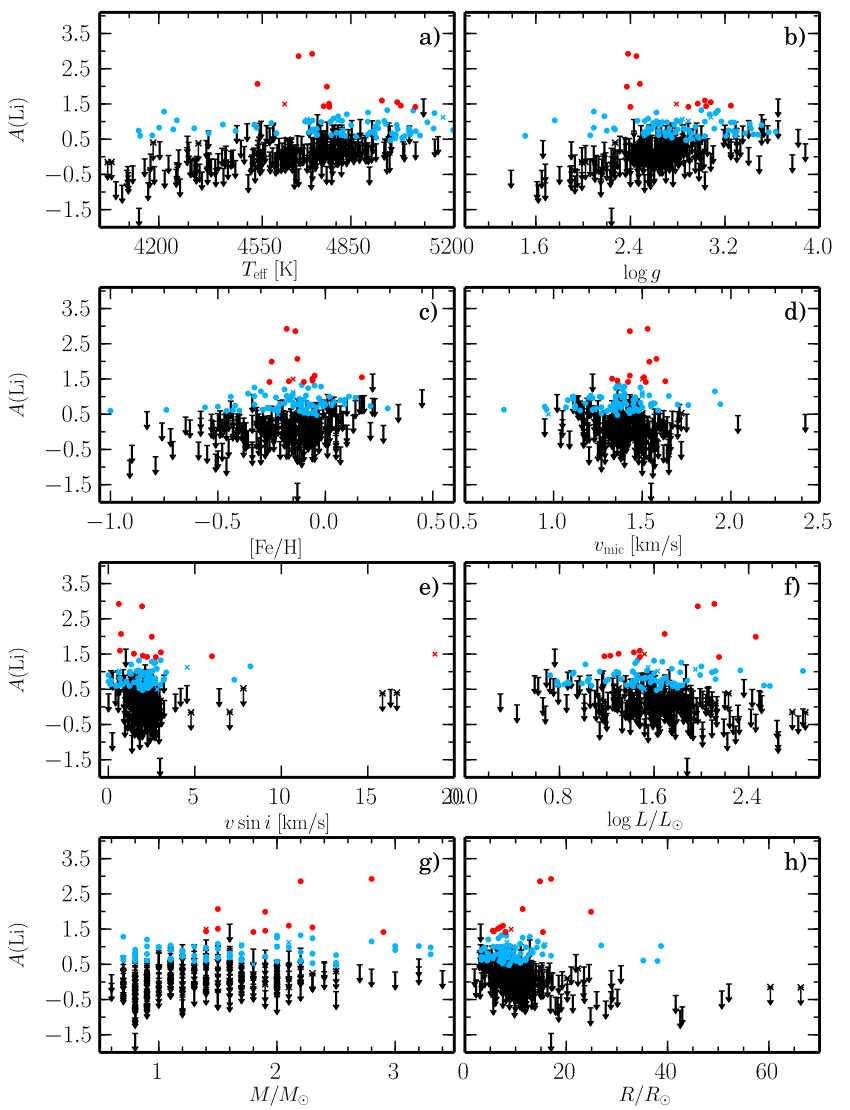

Fig. 8. Lithium abundances vs. available stellar parameters. Color coding is the same as in Fig. 7.

in a particular region of the HR diagram in Fig. 7, and thus, they do not seem to be associated with any particular evolutionary stage.

Interestingly enough, the four most Li-rich giants in our sample form a line on HR diagram, suggesting a similar evolutionary phase; however, the superposition in the HR diagram of the location of stars with different masses and metallicities complicates identification of their evolutionary stage.

\subsection{Lithium abundances versus stellar parameters}

To understand the behavior of the lithium abundances in giants, we checked for their possible relation with the available stellar parameters. Plotted in Figs. $8 \mathrm{a}-\mathrm{h}$ are the $A(\mathrm{Li})$ versus spectroscopic parameters $\left(T_{\text {eff }}, \log g,[\mathrm{Fe} / \mathrm{H}], v_{\text {mic }}, v \sin i\right)$ and estimated integrated parameters (luminosities, masses and stellar radii). The points are color-coded, as in Fig. 7, according to the Li content. The mean and median values, as well as dispersions of the relevant stellar parameters obtained for the three groups, are presented in Table 6.

The relation between $T_{\text {eff }}$ and $A(\mathrm{Li})$ is explored in Fig. 8a, where we see an increase of $A(\mathrm{Li})$ with effective temperature as already shown in Fig. 5. Giants with detected Li practically occupy the whole range of available $T_{\text {eff }}$, while those with enhanced $\mathrm{Li}$ abundance stay within a limited range of $T_{\text {eff }}>$ $4500 \mathrm{~K}$. The four giants with greatest $A(\mathrm{Li})$ all have $T_{\text {eff }}$ in relatively narrow range between 4500 and $4800 \mathrm{~K}(4673 \pm 88 \mathrm{~K}$ on average).

A considerable fraction of stars occupy a narrow range of effective temperatures between 4700 to $5100 \mathrm{~K}$. In this region, two
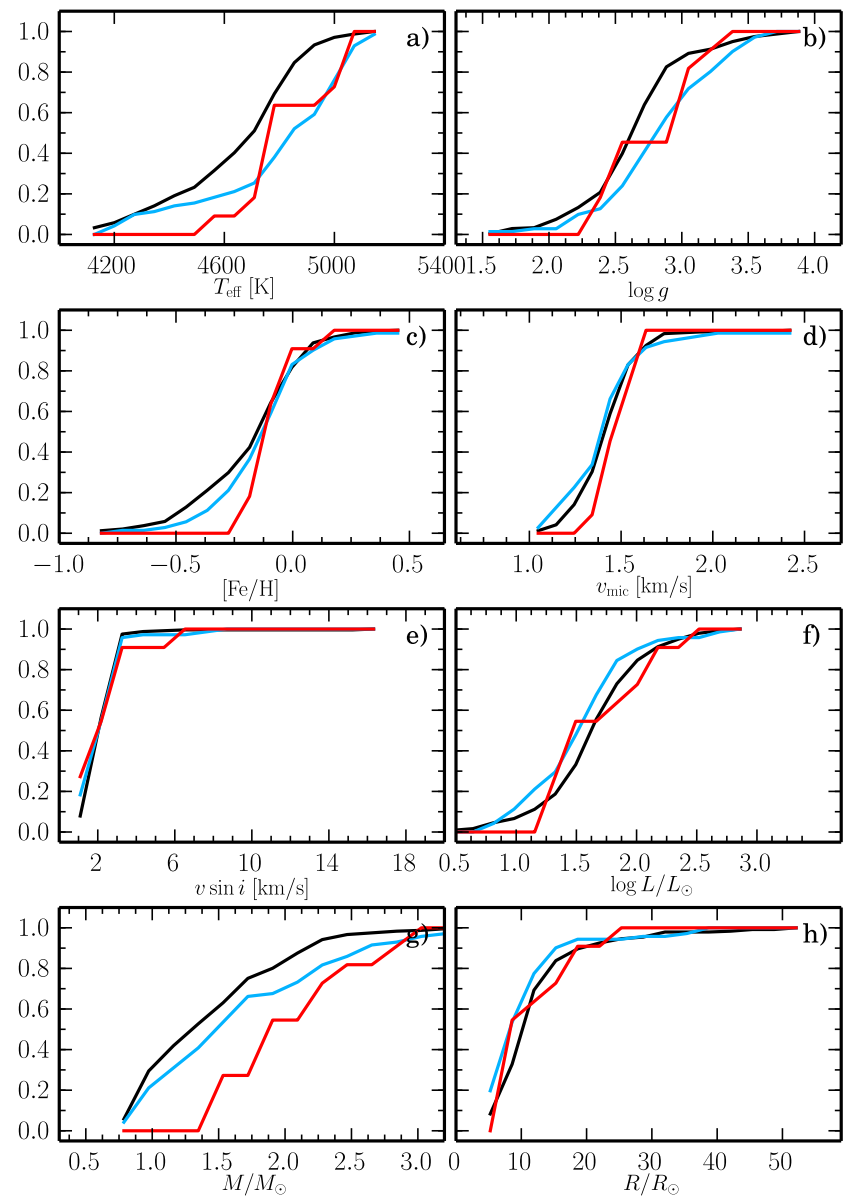

Fig. 9. Cumulative distributions of available stellar parameters. Color coding is the same as in Fig. 7.

different evolutionary stages are expected to be co-existing: giants on their first ascent on RGB that still undergo FDU dilution, and stars in the clump, that already burn helium steadily in their cores. The latter group of stars should reveal lower lithium abundances compared to giants on their way to RGB tip in the absence of an extra-mechanism of Li production. Stars in the clump have already finished depletion due to the FDU, and their lithium abundances were lowered by extra mixing process, which operates after the RGB bump. Although separating those two groups is difficult and requires detailed spectral analysis, stars in the clump are expected to belong to a narrow luminosity range. The $T_{\text {eff }}$ averages for groups A - reveal higher effective temperatures, although within uncertainties for the Li-rich stars (see Table 6).

Figure $8 \mathrm{~b}$ presents a relation between lithium abundance and the surface gravity. Again, giants with detected Li are present at all $\log g$ values. Stars with $A(\mathrm{Li})$ enhanced are placed within a range of $\log g$ between 2.4 and 3.3, while the four objects with the largest $\mathrm{Li}$ overabundances seem to be located in a very narrow range of $\log g$ and, within uncertainties, all four stars have identical $\log g$ of $2.42 \pm 0.05$.

Another interesting feature of Fig. 8b is a significant drop in the number of giants with detected Li for stars with $\log g \lesssim 2.4$. This may be, however, an artifact, which results from the $\log g$ distribution in the sample (cf. Zieliński et al. 2012). Group A giants show the lowest $\log g$ on average; however, no statistically significant difference is found in the mean surface gravity between groups $\mathrm{A}-\mathrm{C}$. 
Table 6. Mean values of stellar parameters obtained for the different subsamples: (Group A) Li-poor giants (non-detections and upper limits), (B) giants with Li measurements, excluding Li-rich stars, and (C) Li-rich stars.

\begin{tabular}{c|ccc|ccc|ccc}
\hline \hline & \multicolumn{3}{|c|}{ A } & \multicolumn{3}{c|}{ B } & \multicolumn{3}{c}{ C } \\
& Median & Mean & $\sigma$ & Median & Mean & $\sigma$ & Median & Mean & $\sigma$ \\
\hline$T_{\text {eff }}$ & 4691 & 4642 & 234 & 4836 & 4790 & 269 & 4776 & 4823 & 159 \\
$\log g$ & 2.64 & 2.62 & 0.40 & 2.79 & 2.81 & 0.42 & 2.89 & 2.76 & 0.32 \\
{$[\mathrm{Fe} / \mathrm{H}]$} & -0.15 & -0.19 & 0.22 & -0.12 & -0.16 & 0.20 & -0.13 & -0.11 & 0.11 \\
$v_{\text {mic }}$ & 1.40 & 1.41 & 0.17 & 1.38 & 1.37 & 0.20 & 1.51 & 1.48 & 0.09 \\
$v \sin i$ & 2.08 & 2.10 & 1.17 & 1.98 & 2.04 & 1.27 & 1.99 & 2.18 & 1.45 \\
$\log L / L_{\odot}$ & 1.62 & 1.63 & 0.42 & 1.52 & 1.52 & 0.43 & 1.48 & 1.68 & 0.41 \\
$M / M_{\odot}$ & 1.30 & 1.40 & 0.55 & 1.50 & 1.62 & 0.69 & 1.90 & 2.03 & 0.48 \\
$R / R_{\odot}$ & 10.00 & 11.68 & 7.35 & 8.20 & 9.88 & 6.97 & 8.00 & 11.24 & 5.87 \\
\hline
\end{tabular}

No obvious relation between $A(\mathrm{Li})$ and $[\mathrm{Fe} / \mathrm{H}]$ is present in Fig. 8c. Li seems to be detected in giants in our sample with all available $[\mathrm{Fe} / \mathrm{H}]$. Those with raised $A(\mathrm{Li})$ have, however, $[\mathrm{Fe} / \mathrm{H}]$ in a range of -0.26 to 0.17 . The four most $\mathrm{Li}$ abundant giants in our sample show practically identical metallicities of $-0.17 \pm 0.05$. Giants with detected Li (group B) and Li-rich giants (group C) seem to have higher metallicities on average (see Table 6), but this might just reflect poor statistics.

In Fig. 8d, we see that giants with detected Li present microturbulence velocities in the whole range available in our sample. The Li-abundant ones, group $\mathrm{C}$, occupy a tighter range of $v_{\text {mic }}=1.3-1.6$, and the four giants with largest $A(\mathrm{Li})$ have $v_{\text {mic }}=1.52 \pm 0.06 \mathrm{~km} \mathrm{~s}^{-1}$, which is identical within uncertainties. No meaningful differences exist between mean values in groups $\mathrm{A}-\mathrm{C}$.

The rotational velocities $v \sin i$ (obtained during SME analysis) versus $A(\mathrm{Li})$ are presented in Fig. 8e. Fast rotators in the RGB are defined as stars with $v \sin i \gtrsim 10 \mathrm{~km} \mathrm{~s}^{-1}$ (Fekel 1997). Most of our stars have rotation velocities expected during the RGB as a consequence of conservation of angular momentum; that is, $v \sin i \approx 2-3 \mathrm{~km} \mathrm{~s}^{-1}$ (Gray 1989; de Medeiros \& Mayor 1999; Fekel 1997). The possible relation of stellar rotation with the overabundance of $\mathrm{Li}$ has been recently explored by Carlberg et al. (2012), where the authors found that rapid rotators are on average enriched in lithium when compared to the slow rotators. All four stars that show the largest amount of lithium have $v \sin i<5 \mathrm{~km} \mathrm{~s}^{-1}$. Moreover, the average rotation velocity of all the Li-detected stars are very similar. The only exception is TYC 0405-01700-1, which is plotted in Fig. 8e with a red cross. With uncertain stellar parameters, this star shows broad line profiles that may by partly attributed to rotation but it shows RV variations of SB2 type at the same time. Its actual status can be resolved with a more detailed spectroscopic analysis, which is beyond the scope of this paper.

With regard to luminosity, Fig. 8f, we note that the giants with $\mathrm{Li}$ detection are present among stars in a wide range of luminosities $\log L / L_{\odot}>0.7$, which practically in all our samples. The giants with enhanced $A(\mathrm{Li})$ all have $\log L / L_{\odot}>1.2$, and the four most $\mathrm{Li}$ abundant ones have $\log L / L_{\odot}$ in 1.6-2.4 range $(2.06 \pm 0.28$ on average $)$.

Giants with Li detection also practically cover the whole range of stellar masses as illustrated in Fig. 8g. The higher the $\mathrm{Li}$ content, the higher the stellar mass, and $\mathrm{Li}$ enhanced giants all show masses larger than $1.4 M_{\odot}$. The most Li abundant giants belong to most massive ones in our sample. The four most $\mathrm{Li}$ abundant giants have $M / M_{\odot}=2.1 \pm 0.5$ on average, and the two most $\mathrm{Li}$ rich have masses of $2-3 M_{\odot}$, which is
Table 7. Kolmogorov-Smirnov tests for stellar parameters: (Group A) Li-poor giants (non-detections and upper limits), (B) giants with Li measurements, excluding Li-rich stars, and (C) Li-rich stars.

\begin{tabular}{c|cc|cc|cc}
\hline \hline & \multicolumn{2}{|c|}{ A vs. B } & \multicolumn{2}{|c|}{ A vs. C } & \multicolumn{2}{c}{ B vs. C } \\
& $\mathrm{D}$ & $\mathrm{p}$ & $\mathrm{D}$ & $\mathrm{p}$ & $\mathrm{D}$ & $\mathrm{p}$ \\
\hline$T_{\text {eff }}$ & 0.37 & 0.00 & 0.39 & 0.06 & 0.27 & 0.43 \\
$\log g$ & 0.26 & 0.00 & 0.37 & 0.08 & 0.31 & 0.25 \\
{$[\mathrm{Fe} / \mathrm{H}]$} & 0.09 & 0.73 & 0.31 & 0.23 & 0.24 & 0.58 \\
$v_{\text {mic }}$ & 0.13 & 0.32 & 0.37 & 0.09 & 0.44 & 0.04 \\
$v \sin i$ & 0.13 & 0.27 & 0.24 & 0.54 & 0.16 & 0.95 \\
$\log L / L_{\odot}$ & 0.17 & 0.08 & 0.22 & 0.62 & 0.24 & 0.60 \\
$M / M_{\odot}$ & 0.15 & 0.14 & 0.53 & 0.00 & 0.41 & 0.06 \\
$R / R_{\odot}$ & 0.24 & 0.00 & 0.29 & 0.28 & 0.27 & 0.45 \\
\hline
\end{tabular}

higher above average. The mean values in groups $\mathrm{A}-\mathrm{C}$ are presented in Table 6 . They support a $A(\mathrm{Li})-$ stellar mass relation (Mallik 1999), although mean values are associated with large uncertainties.

In Fig. 8h, we see that giants with Li detection or overabundance tend to have moderate radii, up to about $39 R_{\odot}$. This is especially true for the four giants with the largest $\mathrm{Li}$ abundance and with radii ranging from 11 to 25 solar radii $\left(17 \pm 5 R_{\odot}\right.$ on average). The lack of Li detection among the giants with the largest radii, however, may very well only reflect the small number of stars from our sample in that range. The average values do not show significant differences among stars for various groups.

In general, we note that the differences in stellar parameters between groups $\mathrm{A}-\mathrm{C}$ are not obvious. However, interestingly enough, the four most $\mathrm{Li}$ abundant giants in our sample (TYC 0684-00553-1, TYC 3105-00152-1, TYC 3304-00090-1, and TYC 3917-01107-1) seem to have very similar stellar parameters, are not fast rotators, and have surprisingly similar $\log g$, metallicity and microturbulence velocity. The largest discrepancies are present in the least constrained ones: masses, radii and luminosities. This is especially true for the two giants with the largest $A(\mathrm{Li})$, TYC 0684-00553-1 and TYC 3105-001521 , with almost identical metallicities, luminosities, masses, and radii. They also have masses at the borderline among solar, intermediate, and moderate radii. With their location on HR diagram (Fig. 7), these results suggest some specific evolutionary phase.

Kolmogorov-Smirnov (K-S) tests on the cumulative distribution function (CDF) of the three groups of stars that are defined according to their lithium content and eight stellar parameters are shown in Fig. 9 and summarized in Table 7, where the D parameter (K-S statistic) and the p-value (probability that the two 
samples are driven from the same distribution) are given for each comparison between pairs.

The cumulative distributions of stellar parameters generally confirm our coarse analysis of Fig. 8. The most striking, statistically significant differences between groups A and B are present in distributions of effective temperatures, $\log g$, and radii. Stars with detected $\mathrm{Li}$ are $148 \mathrm{~K}(<1 \sigma)$ hotter on average, have a larger $\log g$ (by 0.19 , again $<1 \sigma)$, and smaller radii $\left(1.8 R_{\odot}\right.$, again $<1 \sigma)$.

One can see from Fig. 9 and Tables 6 and 7 that the largest, statistically significant differences between samples A and $C$ in CDFs exists in stellar masses. Li-rich giants seem to favor higher stellar masses (by $0.6 M_{\odot}$ or about $1 \sigma$ ). Giants with Li detection (group B) are also less massive than those with Li overabundance (group C).

A less noticeable difference also exists in effective temperature distributions, stars from group $\mathrm{C}$ are $181 \mathrm{~K}$ (about $1 \sigma$ ) hotter. Groups $\mathrm{A}$ and $\mathrm{C}$ also show less prominent discrepancies in $\log g$ and $v_{\text {mic }}$. The CDF for surface gravity shows a gap for $\mathrm{Li}$ rich giants in the range of $\log g \approx 2.5-3$ that may be connected to the substantial drop of $\mathrm{Li}$ abundances present in Fig. 8 around this gravity range, but it can also be a result of a poor statistics in this region.

The largest differences between groups $\mathrm{B}$ and $\mathrm{C}$ are distributions of $v_{\text {mic }}$ and stellar masses. In the case of stellar masses, group B giants show distributions more like group A, while $v_{\text {mic }}$ distributions in groups $\mathrm{A}$ and $\mathrm{B}$ are indistinguishable. The $\mathrm{Li}$ rich giants have the largest $v_{\text {mic }}$ on average, which is larger by $0.11(\sim 1 \sigma)$ than giants with detected Li only.

The two-sample K-S test confirms that all three distributions are the same from a statistical point of view, in the case of metallicity, $v \sin i$, and luminosity.

\subsection{Lithium vs. $\alpha$-elements abundances}

We searched for anomalies in terms of the composition of the $\alpha$-elements for the stars belonging to the three groups, defined according to their Li content. These elements are not produced in this mass range (oxygen the exception) and are certainly not expected to be processed during the RGB evolution of a star.

It has been suggested that the $[\alpha / \mathrm{Fe}]$ trend with $[\mathrm{Fe} / \mathrm{H}]$ is due to the time delay between SN II, which produces alpha elements and iron-peak elements (Arnett 1978; Woosley \& Weaver 1995), and SN Ia, which yields mostly iron-peak with little alpha element production. To check unusual chemical compositions associated to SN II explosions in the sample and whether there is any trace of enhanced $A(\mathrm{Li})$ to be associated with such a deviation, we investigated abundance trends with metallicity for several $\alpha$ elements $(\mathrm{O}, \mathrm{Mg}, \mathrm{Ti}, \mathrm{Ca})$ and for aluminum, sometimes defined as mild- $\alpha$ element. The $[\mathrm{X} / \mathrm{Fe}]$ abundances, as a function of the $[\mathrm{Fe} / \mathrm{H}]$ are shown in Fig. 10. The mean and median values of $[\mathrm{X} / \mathrm{Fe}]$ abundances, as well as dispersions, are presented for all three groups in Table 8. The CDF are presented in Fig. 11, and the results of K-S tests are listed in Table 9.

As it was in the case of stellar parameters, we can see that giants with detected $\mathrm{Li}$ are present in whole range of abundances covered with our sample. Li-rich giants occupy generally a more narrow range, reflecting their metallicities. We see for all elements the expected trend for a lower $[\mathrm{X} / \mathrm{Fe}]$ ratio as the metallicity increases. Around solar metallicities, the relation becomes more flat, which is attributed to the possible onset of SN Ia (see McWilliam 1997). Only few stars stand out from the relation and are those with large uncertainties in their abundances determination or stars rejected from statistical analysis
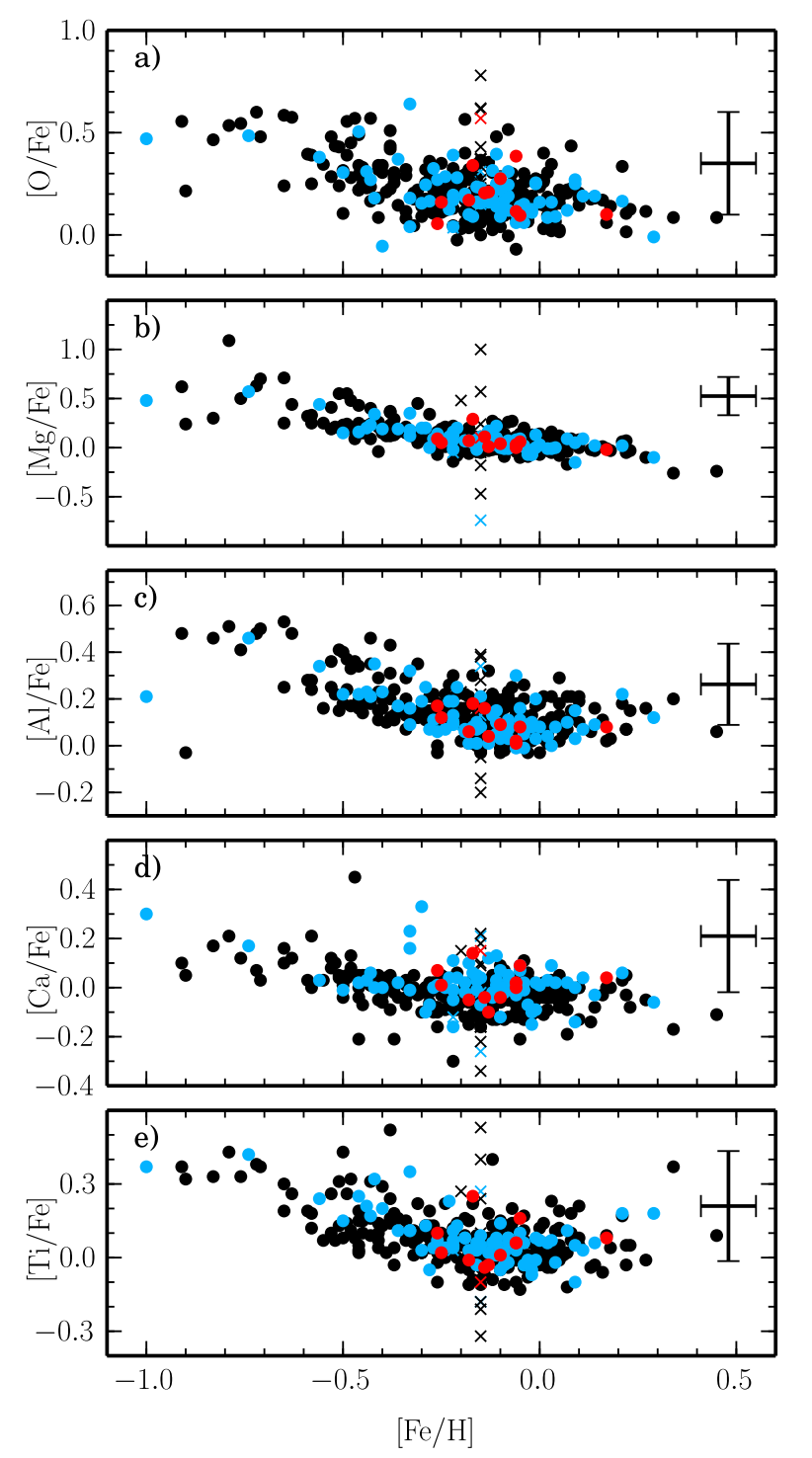

Fig. 10. $[\alpha / \mathrm{Fe}]$ abundances versus metallicity. Color and points coding are the same as in Fig. 7.

due to uncertain stellar parameters or identified as spectroscopic binaries. Lithium rich stars follow the same overall trend with $[\mathrm{Fe} / \mathrm{H}]$ as all the $\alpha$ elements analyzed.

The K-S tests confirm that there are not statistically significant differences between $\alpha$-elements abundances CDF for groups $\mathrm{A}-\mathrm{C}$. In the case of aluminum and magnesium, we note a systematic effect: the giants with $\mathrm{Li}$ detection or overabundance show lower values of $[\mathrm{Al} / \mathrm{Fe}]$ and $[\mathrm{Mg} / \mathrm{Fe}]$, however, at the level well below $1 \sigma$. We find no statistically significant trace of $\alpha$-elements enhancement in giants with detectable Li content. The only outlier is TYC 0405-1700-1, which presents slight $\mathrm{O}$ enhancement.

\subsection{Li overabundance and signatures of mass loss}

If planet engulfment was the cause of lithium abundance anomalies, then it would be reasonable to expect a relation of lithium abundance and potential mass ejections from the star (see e.g. de La Reza et al. 1997; Siess \& Livio 1999; Lebzelter et al. 2012) that might show as infrared excesses. We have collected data from WISE (Wright et al. 2010), IRAS (Neugebauer et al. 1984), and 2MASS catalogs for the stars in our sample in search 
M. Adamów et al.: The Penn State - Toruń Centre for Astronomy Planet Search stars. II.

Table 8. Mean values of abundances obtained for the different subsamples: (Group A ) Li-poor giants, (B) giants with Li measurements, excluding Li-rich stars, and (C) Li-rich stars.

\begin{tabular}{c|ccc|ccc|cccc}
\hline \hline & \multicolumn{3}{|c|}{ A } & \multicolumn{3}{c|}{ B } & \multicolumn{3}{c}{ C } \\
& Median & Mean & $\sigma$ & Median & Mean & $\sigma$ & Median & Mean & $\sigma$ \\
\hline$[\mathrm{O} / \mathrm{Fe}]$ & 0.19 & 0.21 & 0.13 & 0.18 & 0.21 & 0.12 & 0.17 & 0.19 & 0.10 \\
{$[\mathrm{Mg} / \mathrm{Fe}]$} & 0.08 & 0.11 & 0.16 & 0.07 & 0.09 & 0.12 & 0.05 & 0.07 & 0.08 \\
{$[\mathrm{Al} / \mathrm{Fe}]$} & 0.13 & 0.15 & 0.11 & 0.09 & 0.12 & 0.09 & 0.08 & 0.09 & 0.06 \\
{$[\mathrm{Ca} / \mathrm{Fe}]$} & -0.02 & -0.02 & 0.08 & 0.01 & 0.02 & 0.09 & 0.01 & 0.01 & 0.07 \\
{$[\mathrm{Ti} / \mathrm{Fe}]$} & 0.06 & 0.08 & 0.11 & 0.06 & 0.08 & 0.10 & 0.06 & 0.06 & 0.08 \\
\hline
\end{tabular}
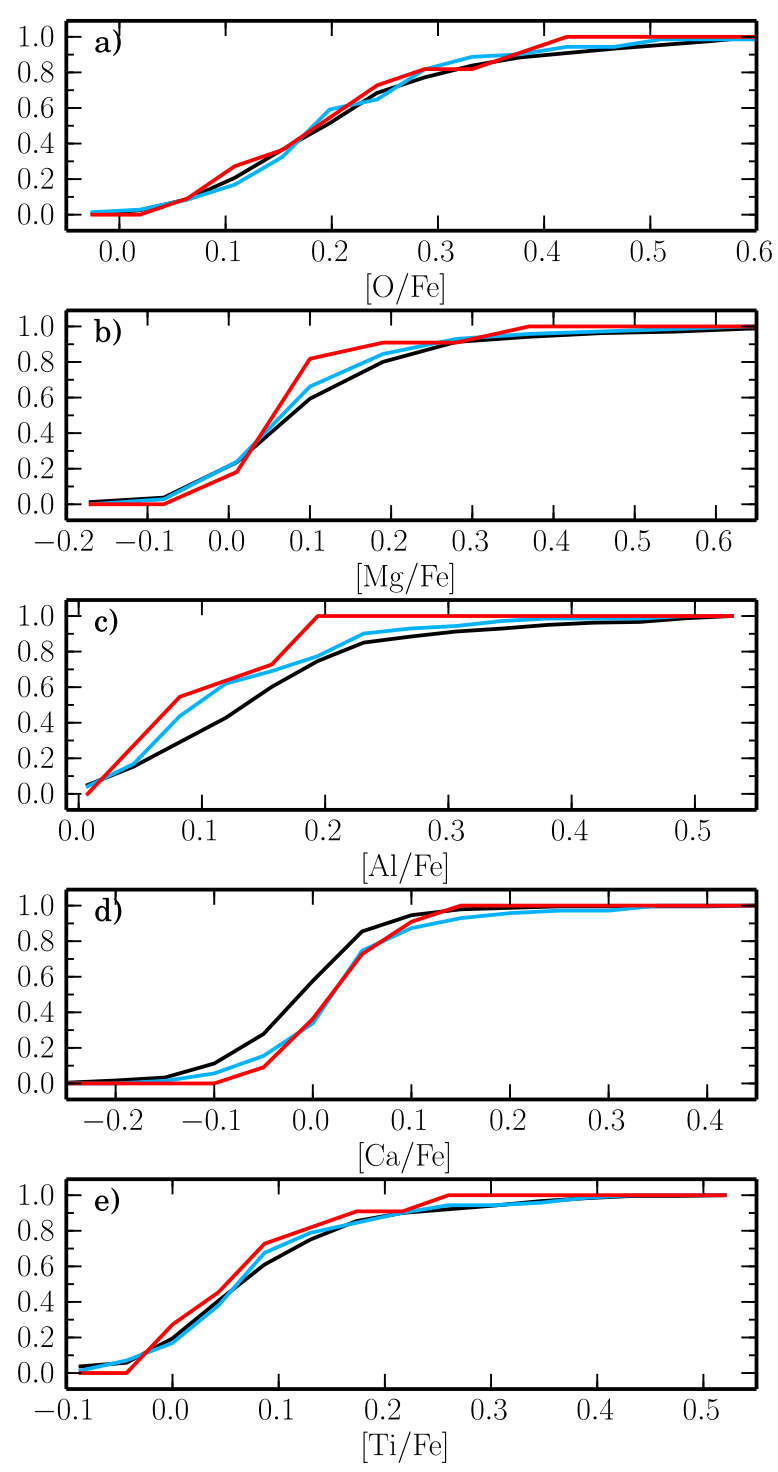

Fig. 11. Cumulative distributions of $[\mathrm{X} / \mathrm{Fe}]$ values. Color coding is the same as in Fig. 7.

for infrared excesses. A total of 317 stars from the 323 sample, 16 giants with uncertain parameters, and two spectroscopic binaries were identified in the WISE catalog with only four stars falling outside of the $1^{\prime \prime}$ search radius and 83 matches within a $25^{\prime \prime}$ search radius were found in the IRAS catalog (queried in both the Point Source Catalog and Faint Source Catalog).

In Fig. 12, we present the $K$-WISE[12 $\mu \mathrm{m}]$ color versus the $J-K$ 2MASS color diagram of our stars, based on the color selection presented by Lebzelter et al. (2012), who investigated
Table 9. Kolmogorov-Smirnov tests for abundances: (Group A) Li-poor giants; (B) giants with Li measurements, excluding Li-rich stars, and (C) Li-rich stars.

\begin{tabular}{c|cc|cc|cc}
\hline \hline & \multicolumn{2}{|c|}{ A vs. B } & \multicolumn{2}{|c|}{ A vs. C } & \multicolumn{2}{c}{ B vs. C } \\
& D & p & D & p & D & p \\
\hline$[\mathrm{O} / \mathrm{Fe}]$ & 0.10 & 0.62 & 0.27 & 0.37 & 0.21 & 0.74 \\
{$[\mathrm{Mg} / \mathrm{Fe}]$} & 0.08 & 0.83 & 0.24 & 0.51 & 0.20 & 0.81 \\
{$[\mathrm{Al} / \mathrm{Fe}]$} & 0.13 & 0.29 & 0.29 & 0.30 & 0.21 & 0.74 \\
{$[\mathrm{Ca} / \mathrm{Fe}]$} & 0.10 & 0.58 & 0.21 & 0.68 & 0.22 & 0.69 \\
{$[\mathrm{Ti} / \mathrm{Fe}]$} & 0.10 & 0.67 & 0.24 & 0.54 & 0.22 & 0.69 \\
\hline
\end{tabular}

the possibility of enhanced mass-loss in lithium rich stars. Only few stars, including one Li-rich star (TYC 0405-01700), are outliers from the general flat relation $K$-WISE[12 $\mu \mathrm{m}]=0.06$. However, with $K$-WISE[12 $\mu \mathrm{m}]<1$ they do not appear to be objects with substantial mass loss taking place (Lebzelter et al. 2012).

Figure 13 shows an IRAS two color-color diagram with three regions defined by de La Reza et al. (1997) based on mass loss rates and the IR excess consequence of it. In the region labeled as I no Li-rich nor fast rotating giants are expected as, according to de La Reza et al. (1997), in this region only "normal" $\mathrm{K}$ giants, i.e. stars with low lithium abundances and no signatures of circumstellar shell should be present. Region II delimitates a color-color region with infrared excesses. If lithium production was associated to a planet engulfment episode in the form of a mass loss increase then the star should move to region II where infrared excess is observed. When this episode ends, the star should move to region III, where a small infrared excess is still present, and finally will head back to region I closing the cycle.

Region I is poorly populated - only two stars are located in here - TYC 3226-02285-1 and TYC 3930-01790-1, one with detected $\mathrm{Li}$. These are also the only two stars with both IRAS colors available. For all remaining stars only upper limits in one or both IRAS colors are available. In the most interesting region II we find 10 stars, all with upper limits of Li abundances. The vast majority of the stars identified in the IRAS catalog, 69 out of $83(83 \pm 4 \%)$, are located in region III. In that region we identified super-Li star TYC 3917-01107-1, 11 stars with detected Li (group B) and 58 from group A. Only $16 \pm 5 \%$ of stars in that region belong to group $\mathrm{B}$ or $\mathrm{C}$.

What Fig. 13 seems to imply is that most of giants identified in IRAS catalog have moderate infrared excesses. These excesses, if triggered by an engulfment process, would suggest that it has happened recently, and we observe stars before their envelopes became diluted. It seems more reasonable to assume that if the nature of the excess is real, then it is most likely associated with RGB mass loss than due to an extra mechanisms. 


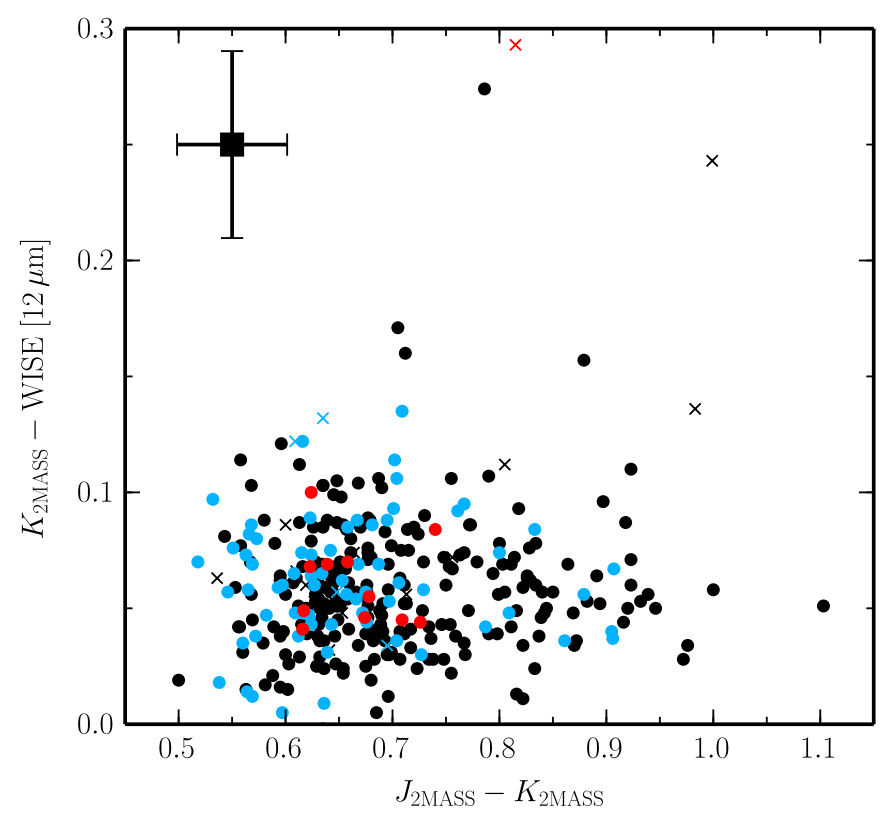

Fig. 12. $K$-WISE[12 $\mu \mathrm{m}]$ color - versus the $J-K$ 2MASS color diagram. The outlier in the $K$-WISE[12 $\mu \mathrm{m}$ ] color is TYC 0405-01700-1. Color coding is the same as in Fig. 7.

Another empirical way of investigating the mass loss phenomena in our sample is through the $\mathrm{Na} \mathrm{D}$ lines profiles. In cases of intense mass loss, an additional component in the sodium lines should be observed on the blue side of the $\mathrm{Na}$ structure and should be separated from the stellar line from several up to a dozen $\mathrm{km} \mathrm{s}^{-1}$ (Reddy et al. 2002). The problem with the $\mathrm{NaD}$ line features in our spectra is that the data reduction process needs to be done extremely carefully due to possible contamination of the stellar spectra by emission structures in the flat-field lamp. This spectral range requires also removal of the iodine lines (which is a part of RV code for line bisector calculations) but introduces extra noise. We have performed this exercise but only for the stars that belong to the Li-rich sample (group C). Complicated structures around $\mathrm{NaD}$ lines are present for some stars. For example, TYC 3663-01966-1 presents additional components on the blue side of spectra. However, we found it difficult to identify their origin and assign it to circumstellar gas instead of to the interstellar medium.

Intensive mass outflows might also have the effect of changing the shape of observed spectral lines, a phenomena that should be observable in a few years span. Since PTPS data has been collected since 2004, we checked for differences in the line shape of selected stars. We analyzed the sample of Li-rich stars, and we did not find any significant differences in the line shapes that could be attributed to mass loss.

\section{Analysis of the individual stars that present Li overabundances}

In this section, we present detailed, individual comments of the 12 giant stars that show high Li abundances (group $\mathrm{C}$ and TYC 0405-01700-1). Their parameters are listed in Table 10.

\subsection{Super-Li giants}

The object TYC 0684-00553-1 with $A(\mathrm{Li})>3.3$ is the star with the largest Li content identified in this work. Its Li abundance is

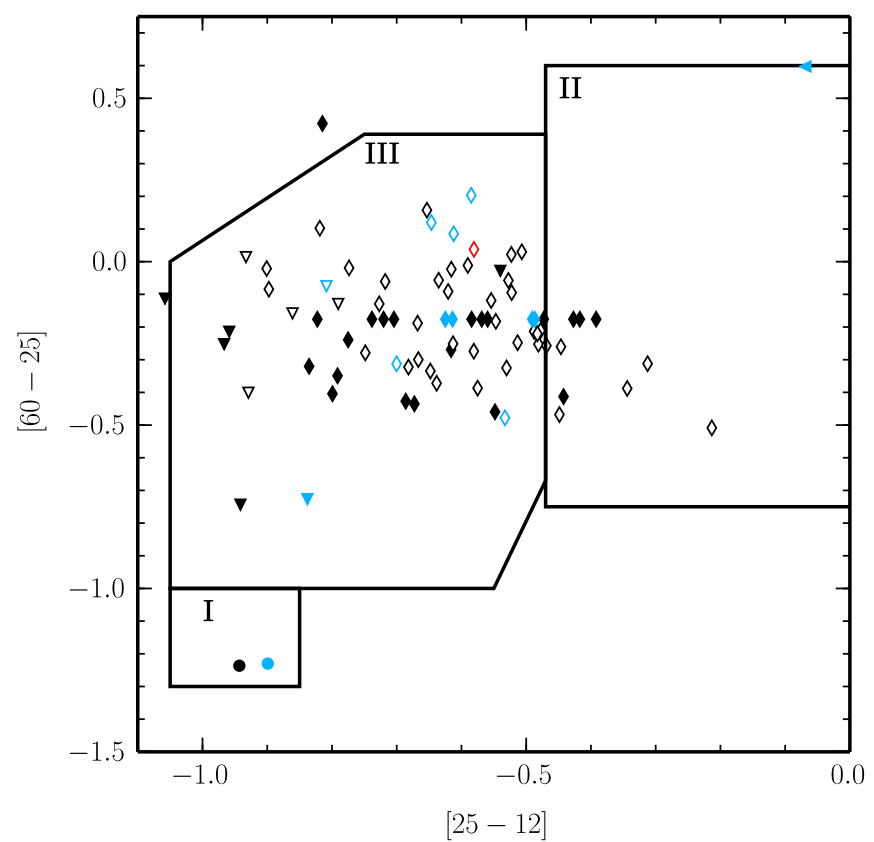

Fig. 13. The Li-cycle diagram according to de La Reza et al. (1997). Filled and open symbols represent different IRAS catalogs: PSC - Point Source Catalog, FSC - Faint Source Catalog, respectively. Color coding is the same as in Fig. 7. Symbols stand for IRAS fluxes quality: circles - both colors have good quality, triangles pointing left - upper limit for [25-12], triangles pointing down - upper limit for [60-25], diamonds - upper limits for both colors.

close to the meteoritic value, so it is clear that a Li-enhancement process must have been operating in this star. With the exception of the high $A(\mathrm{Li})$, we found no signs of any enrichment processes: this star shows normal abundances of other elements studied here, slow rotation, and no presence of IR excess in WISE photometry (no matching identification was found in the IRAS catalog). With a mass of $2.8 M_{\odot}$, this star does not undergo a luminosity bump on the RGB, and regarding its location in the HR diagram, it might be in the clump or post clump phase. The HET/HRS radial velocities collected so far for TYC 0684-00553-1 reveal chaotic changes with an amplitude of $\sim 100 \mathrm{~m} \mathrm{~s}^{-1}$, which exclude a stellar companion, but give no constraints on planetary companion, stellar activity, or pulsations yet.

The star TYC $3105-00152-1$ is very similar to TYC 068400553-1 in terms of effective temperature ( $\left.T_{\text {eff }}=4673 \mathrm{~K}\right), \log g$, and metallicity. It is also the second giant star with a $\mathrm{Li}$ abundance close to meteoritic value identified in this work. With a mass of $2.2 M_{\odot}$ it is expected, unlike TYC 0684-00553-1, to experience the luminosity bump on the RGB. For $2.2 M_{\odot}$ stars with solar metallicity, the LFB occurs at $T_{\text {eff }} \sim 4400 \mathrm{~K}$ (Charbonnel $\&$ Balachandran 2000). Although our luminosity and masses are uncertain, the uncertainty in $T_{\text {eff }}$ is very small $(15 \mathrm{~K}$, determined by Zieliński et al. 2012), and the star does not seem to be associated with the LFB. Precise HET/HRS RV for this star reveal an amplitude of $\sim 100 \mathrm{~m} \mathrm{~s}^{-1}$, which, again, seem to exclude a stellar companion but are not numerous enough for a detailed analysis yet.

The object TYC 3304-00090-1 = BD+48 740 with $A(\mathrm{Li})=$ 2.2 and a radial velocity amplitude of $70 \mathrm{~m} \mathrm{~s}^{-1}$ was analyzed in detail by Adamów et al. (2012). This is the third most Li-rich giant in our sample, which is an interesting star hosting a planet. The $\mathrm{Li}$ abundance analysis has been extended with new epochs 
M. Adamów et al.: The Penn State - Toruń Centre for Astronomy Planet Search stars. II.

Table 10. Stellar parameters for the 11most Li abundant giants in our sample and TYC 0405-01700.

\begin{tabular}{|c|c|c|c|c|c|c|c|c|c|c|}
\hline TYC & $\begin{array}{l}T_{\text {eff }} \\
{[\mathrm{K}]}\end{array}$ & $\mathrm{Fe} / \mathrm{H}$ & $\begin{array}{l}A(\mathrm{Li}) \\
\text { NLTE }\end{array}$ & $\begin{array}{c}v \sin i \\
{\left[\mathrm{~km} \mathrm{~s}^{-1}\right]}\end{array}$ & $\log L / L_{\odot}$ & $\begin{array}{c}M \\
{\left[M_{\odot}\right]}\end{array}$ & $\begin{array}{r}R \\
{\left[R_{\odot}\right]}\end{array}$ & RV status & $\begin{array}{l}\text { Infrared } \\
\text { photometry }\end{array}$ & Comment \\
\hline 0684-00553-1 & 4719 & -0.18 & 2.92 & 0.6 & 2.11 & 2.8 & 17.0 & RV var & - & \\
\hline $3105-00152-1$ & 4673 & -0.14 & 2.86 & 2.0 & 1.97 & 2.2 & 14.8 & RV var & - & \\
\hline 3304-00090-1 & 4534 & -0.13 & 2.07 & 0.7 & 1.69 & 1.5 & 11.4 & planet & & Adamów et al. (2012) \\
\hline 3917-01107-1 & 4769 & -0.25 & 1.99 & 2.5 & 2.46 & 1.9 & 24.9 & planet & IRAS, III & Niedzielski et al. (in prep.) \\
\hline 0435-03332-1 & 4955 & -0.05 & 1.60 & 0.7 & 1.48 & 2.1 & 7.5 & single & - & \\
\hline $1058-02865-1$ & 4758 & -0.17 & 1.44 & 6.0 & 1.18 & 1.4 & 5.7 & single & - & \\
\hline $3300-00133-1$ & 5007 & 0.17 & 1.55 & 3.0 & 1.43 & 2.3 & 6.9 & single & - & \\
\hline 3314-01371-1 & 5019 & -0.06 & 1.45 & 2.0 & 1.23 & 1.9 & 5.5 & SB1 & - & \\
\hline 3318-01333-1 & 4776 & -0.06 & 1.51 & 1.5 & 1.30 & 1.5 & 6.5 & RV var & - & \\
\hline 3663-01966-1 & 5068 & -0.26 & 1.41 & 2.7 & 2.15 & 2.9 & 15.4 & single & - & \\
\hline 3930-00681-1 & 4777 & -0.10 & 1.42 & 2.2 & 1.48 & 1.8 & 8.0 & single & - & \\
\hline 0405-01700-1 & $(4626)$ & $(-0.15)$ & $(1.50)$ & $(18.9)$ & $(1.52)$ & $(1.4)$ & $(9.1)$ & SB2 & WISE & est. stellar params. \\
\hline
\end{tabular}

of HET/HRS observations, so we present an updated lithium abundance of $A(\mathrm{Li})=2.07$ here. In the HR diagram this star is located near LFB, however its $\log L / L_{\odot}$ is uncertain. The preliminary determined eccentric orbit of the observed planet with the Li enrichment suggest a recent engulfment of a putative second planet as a very plausible scenario. However, a slow rotation rate and the absence of IR excess do not support this conclusion. An extensive analysis of this system is in preparation.

The giant TYC 3917-01107-1, a $1.9 M_{\odot}$ star is another example of a planetary system around a Li-rich giant (Niedzielski et al., in prep.). This is the fourth most Li-rich star in our sample. In spite of large uncertainties in luminosity $\left(\log L / L_{\odot}=\right.$ $2.46 \pm 0.6$ ), the star seems to be located above the clump region. With the effective temperature of $T_{\text {eff }}=4769 \mathrm{~K}$, it is unlikely that it is currently undergoing LFB (for $2 M_{\odot}$, LFB occurs at $\left.T_{\text {eff }} \sim 4400 \mathrm{~K}\right)$. Thus, lithium enrichment does not seem to be an effect of Li production during LFB. TYC 3917-01107-1 is the only super-Li giant in our sample with IRAS data that point to a trace of IR excess, which may be a result of mass loss and makes engulfment scenario more likely.

\subsection{Li-rich giants}

The star TYC 0435-03332-1 is located on the HR diagram in the RGC region; however we cannot rule out the possibility that it is a giant in the early RGB with ongoing Li dilution caused by the FDU. Based on stellar evolutionary tracks and observed Li abundances for MS and subgiant stars (Ramírez et al. 2012; Mallik 1999), we can assume that this star left MS with $A(\mathrm{Li}) \sim$ 2-2.5. The current Li level suggests a dilution by up to 1 dex. Only a few epochs of HET/HRS RVs, are available for this star as it was classified in PTPS as a single.

The object TYC 1058-02865-1 has a low luminosity $\left(\log L / L_{\odot}=1.18\right)$, solar-mass $\left(1.4 M_{\odot}\right), T_{\text {eff }}=4758$ and $A(\mathrm{Li})=1.4$. This star should have left the MS with $A(\mathrm{Li}) \sim 2$, and it is most likely still undergoing the FDU process. In PTPS, after four epochs of HET/HRS RVs that reveals an amplitude below $50 \mathrm{~m} \mathrm{~s}^{-1}$, it was classified as a single star. Compared to other stars in the group of Li-rich objects, it reveals a relatively fast rotation velocity $\left(6 \mathrm{~km} \mathrm{~s}^{-1}\right)$.

The star TYC 3300-00133-1 has moderate LTE Li abundance of $A(\mathrm{Li})=1.25$ but becomes Li-rich after adding the non-LTE correction of 0.18 . It is located in RGC region, but it is also quite massive $\left(M=2.3 M_{\odot}\right)$, so it cannot be excluded that this star is still undergoing the FDU dilution. In PTPS, it was classified as a single.

The giant TYC 3314-01371-1 is not an RGC object because of its low luminosity and mass of $1.9 M_{\odot}$; thus lithium depletion in the FDU might be still taking place. After 15 epochs of HET/HRS, this star has revealed an RV amplitude above $7 \mathrm{~km} \mathrm{~s}^{-1}$. A preliminary Keplerian fit (Table 11) shows that we are dealing with a subsolar minimum mass companion of $0.24 M_{\odot}$ in a long period, 845 days, with eccentric $(e=0.34)$ orbit. No trace of additional line system was found in our $\mathrm{CCF}$ analysis, so the star was classified as SB1 in PTPS. In addition to quite high lithium abundance, we observed a slightly elevated oxygen abundance, suggesting external enrichment, and no clues for mass transfer.

The object TYC 3318-01333-1 is another $1.5 M_{\odot}$ star, which is not in the RGC because of its low luminosity $\left(\log L / L_{\odot}=1.3\right)$. Therefore, it is probably depleting lithium in FDU. Collected HET/HRS RVs show low amplitude, non-periodic variations.

The star TYC 3663-01966-1 is the most massive star $\left(2.9 M_{\odot}\right)$ in our Li-rich sample. With $\log L / L_{\odot}$ of 2.15 , it is located above RGC, which is close to the early AGB. The $A(\mathrm{Li})_{\mathrm{NLTE}}$ of 1.41 and the observation, that stars of that mass leave the MS with $A(\mathrm{Li}) \sim 3$, suggest that a significant depletion process has already occurred. Another interesting property of this object is its Na profile, which reveals a complicated structure with four non-stellar components on the blue side of the stellar lines. They possibly originate in a circumstellar environment and may be associated with intensive mass loss phenomenon. However, considering that observed lithium abundance is probably due to FDU dilution, intensive mass loss should not be address to $\mathrm{Li}$ enhancement process. Radial velocities collected within PTPS show chaotic, low amplitude variations that cannot be clearly associated with a companions or periodic stellar activity, and the star was assumed to be single.

The giant TYC 3930-00681-1 is located in the RGC region $\left(T_{\text {eff }}=4777 \mathrm{~K}, \log L / L_{\odot}=1.48\right)$. With a mass of $1.8 M_{\odot}$, this star should have left the MS with $A(\mathrm{Li})$ close to 2 . It cannot be excluded that the observed $A(\mathrm{Li})=1.42$ is due to the FDU depletion that has not been completed yet. After a few epochs of precise HET/HRS RV, this star was assumed as single.

\subsection{TYC 0405-01700-1}

This star belongs to the group of 16 giants for which only approximate stellar parameters are available in Zieliński et al. (2012), but it reveals several interesting characteristics. Seven 
Table 11. Preliminary orbital parameters of Li-abundant giants in binary systems.

\begin{tabular}{lll}
\hline \hline Star & TYC 0405-01700-1 & TYC 3314-01371-1 \\
\hline$P[$ days $]$ & $31.753 \pm 0.0009$ & $844.6 \pm 0.6$ \\
$T_{0}[\mathrm{MJD}]$ & $53186.6 \pm 0.45$ & $54498.90 \pm 0.82$ \\
$K\left[\mathrm{~km} \mathrm{~s}^{-1}\right]$ & $28.88 \pm 0.18$ & $3.686 \pm 0.013$ \\
$e$ & $0.040 \pm 0.004$ & $0.3392 \pm 0.0034$ \\
$\omega[\mathrm{deg}]$ & $315 \pm 5$ & $58.7 \pm 0.5$ \\
$m_{2} \sin i\left[M_{\odot}\right]$ & $0.53 \pm 0.08$ & $0.235 \pm 0.022$ \\
$a[\mathrm{AU}]$ & $0.220 \pm 0.016$ & $2.17 \pm 0.08$ \\
$V_{0}\left[\mathrm{~km} \mathrm{~s}^{-1}\right]$ & $-10.59 \pm 0.12$ & $0.758 \pm 0.009$ \\
$\sqrt{\chi_{v}^{2}}$ & 0.80 & 0.92 \\
$\sigma_{\mathrm{RV}}\left[\mathrm{m} \mathrm{s}^{-1}\right]$ & 84.72 & 21.80 \\
$N_{\text {obs }}$ & 7 & 15 \\
\hline
\end{tabular}

epochs of HET/HRS RV measurements available for this star have shown an amplitude of over $40 \mathrm{~km} \mathrm{~s}^{-1}$; therefore, it was classified in the PTPS as a binary. Based on preliminary Keplerian orbital parameters (Table 11), we estimated an orbital period of 31.7 days, which agrees with Kiraga (2012), who classify the star as RS CVn. We also estimate the minimum mass of the companion to $0.53 M_{\odot}$ and postulate that the less massive companion is a more evolved star.

The CCF analysis shows a distortion in the spectral lines, which might be due to an unresolved, second set of lines from other object; thus, all parameters coming directly from spectral analysis should be considered very uncertain. This star seems to be a fast rotator with $v \sin i=19 \mathrm{~km} \mathrm{~s}^{-1}$. It shows the reddest color in WISE and 2MASS photometry, and besides the high $\mathrm{Li}$ abundance, it also shows oxygen enhancement for its adopted metallicity. It is interesting to note that TYC 040501700-1 was identified as a 2MASS object that coincides with a Rosat X-ray source (Haakonsen \& Rutledge 2009), and it might exhibit coronal activity. In such a case, the observed Li enhancement might stem from mass transfer from an evolved stellar companion, which would explain the abnormal chemical composition of TYC 0405-01700-1, its fast rotation, and its mild IR excess. Low eccentricity of the system and a very short period, resulting in orbital separation of only $\sim 5$ stellar radii seem to agree with this scenario. We note, however, that the current secondary mass is only $0.75 M_{\odot}$ if we assume average value of $\sin i$, and the system must have lost/transferred at least $0.65 M_{\odot}$. On the HR diagram, this star is located near the luminosity function bump.

\section{Discussion}

We analyzed the high resolution spectra of a sample of 348 stars in terms of their lithium content. for which we already had accurate determination of stellar parameters from Zieliński et al. (2012). The stars represent a subsample of the 1000 stars being monitored as part of the PTPS program (Niedzielski \& Wolszczan 2008), and thus additional information regarding putative companions is available to us. The sample of Zieliński et al. (2012) is composed mainly of regular giant stars evolving along the RGB $(62 \pm 3 \%)$ and $37 \pm 3 \%$ giants (126 stars) that belong to the RGC according to extended criteria in luminosity to account for uncertainties presented by Zieliński et al. (2012). It is suitable in searching for any lithium enrichment association to a particular evolutionary stage on the RGB.
After rejecting five dwarfs, 16 stars with uncertain stellar parameters, two SB2 systems and two, subgiants from the Zieliński et al. (2012) sample, we performed further analysis of 323 giants with spectroscopically determined parameters and multiple HET/HRS spectra.

We detect a lithium line in 82 out of the 323 stars analyzed ( $25 \pm 2 \%$ of the sample); 11 of these stars are Li-rich according to the criteria that $A(\mathrm{Li})_{\text {NLTE }} \gtrsim 1.4 \mathrm{dex}$, which represents $13 \pm 4 \%$ of the stars with lithium detection (or $3.4 \pm 1 \%$ of the stars in the full sample). Seven stars $(2.2 \pm 1 \%)$ reveal Li abundances above the commonly accepted threshold of $A(\mathrm{Li})>1.5$. We also identify a group of four $(1.4 \pm 1 \%$ of the sample $)$ superLi giants with $A(\mathrm{Li})>2.0$ (within uncertainties), which share many characteristics, such as high mass, luminosity, effective temperature, and metallicity, and are all RV variable. These giants include TYC 0684-00553-1 and TYC 3105-00152-1, stars with $\mathrm{Li}$ abundances close to meteoritic level, and TYC 330400090-1 and TYC 3917-01107-1, giants with $A(\mathrm{Li}) \approx 2$ and planetary systems.

We have located these stars on the HR diagram, correlated their properties in terms of lithium content with the stellar parameters in the search for trends, performed accurate abundance analysis of $\alpha$ elements, looked for infrared excesses, and finally contextualized the Li-rich sample within the additional information provided, since these stars are part of the PTPS program.

Within the narrower range of stellar effective temperatures for the stars in our sample, we find similar behavior in terms of lithium abundances and $T_{\text {eff }}$, as it has been already pointed out in the literature (Brown et al. 1989; Lebzelter et al. 2012; Gonzalez et al. 2009), which is lower in lithium abundances for cooler stars. We have divided the sample in three groups: one, containing all the stars where we provide upper limits for $\mathrm{Li}$ abundance; the second, composed of those stars with $\mathrm{Li}$ detection but $A(\mathrm{Li})_{\mathrm{NLTE}} \lesssim 1.4 \mathrm{dex}$; and a third, containing all the Li-rich giants of the sample.

Regarding the stellar parameters, we find no substantial deviation in the cumulants of different groups in terms of their distribution of luminosity, metallicity, abundances of $\mathrm{Al}, \mathrm{O}, \mathrm{Mg}, \mathrm{Ti}$, and $\mathrm{Ca}$, and rotational velocities. We find, however, differences in effective temperature, gravitational acceleration, microturbulence velocity, stellar mass, and radii. Giants with detected Li favor higher effective temperatures and gravitational accelerations but lower radii. Li-rich giants tend to have even higher effective temperatures and larger masses compared to giants with no lithium, and they have larger $v_{\text {mic }}$ than giants with detected $\mathrm{Li}$. It is important to note, however, that for most of the stars we do not have reliable parallaxes, and therefore, we have an important source of uncertainty in their masses, radii, and luminosities.

The most common scenario for lithium production in giants is the Cameron-Fowler mechanism. It is expected to operate in particular locations on the HR diagram that are usually defined as the RGC or LFB, where extra-mixing is expected to take place. The LFB occurs on the RGB for star with masses $<2.2 M_{\odot}$, which probably has a dependency with metallicity, and takes place no earlier than $T_{\text {eff }} \gtrsim 4400 \mathrm{~K}$ (Charbonnel \& Balachandran 2000).

Thirty-five $(43 \pm 5 \%)$ out of 82 giants with detected Li fall into RGC and only $28(9 \pm 2 \%)$ into LFB. Most of the 11 giants with the largest $\mathrm{Li}$ content and luminosities of $\log L / L_{\odot}=1-1.5$ are likely first ascent RGB stars that will ignite He core fusion at the tip of RGB. Only four of them $(36 \pm 15 \%)$ fall into RGC and one $(9 \pm 9 \%)$ into LFB.

Within uncertainties, these fractions agree with the complete sample, and we conclude that Li-detected giants are distributed 
on HRD, as a complete sample with no preference toward RGC or LFB. The location of the Li-rich stars of our sample,therefore, agrees with the general conclusions of Anthony-Twarog et al. (2013), Martell \& Shetrone (2013), and Lebzelter et al. (2012), who find that Li-rich giants can be found almost anywhere along the RGB and that they are not particularly associated to a particular location on the HR diagram.

On the other hand, the LFB is nothing but the place where an additional mechanism of mixing, which is not defined in standard stellar evolution theory, takes place. Eggleton et al. (2008) showed that the removal of the molecular weight discontinuity is responsible for the observed changes in abundances of various elements, such as in the decline of the ratio of carbon isotopes and the enhancement in nitrogen abundance. According to the recent model of extra mixing driven by fast internal rotation by Denissenkov (2012), abundances of aluminum might be also a clue for extra mixing process. However, we do not see any trends in aluminum abundances for Li-rich stars, which may be associated with extra mixing. Observed statistical differences in $[\mathrm{Al} / \mathrm{Fe}]$ distributions for Li-poor, detectable, and rich stars might reflect that both $\mathrm{Li}$ moderate and rich stars cluster in narrow $[\mathrm{Fe} / \mathrm{H}]$ range.

External pollution from the type II supernova as the origin of the Li-enrichment should reveal a correlation with the $\alpha$-element abundances. We find no conclusive systematic correlation between the abundances of the $\alpha$-elements and the groups established that regards the presence of lithium, although there is the exception of calcium and titanium. If anything, Li-detected stars in our sample seem to show a preference toward lower $\alpha$-element abundances; however, according to the Kolmogorov-Smirnov tests, this relation is not very strong. Furthermore, oxygen and magnesium seem to be less abundant for stars, where lithium can be measured, regardless of the lithium abundance level. If that is a consequence of the inhomogeneous stellar populations present in our sample, it is difficult to address with the data at hand and the lack of accurate distances for most of the stars. There is no sign that Li overabundance is connected to supernovae explosions in neither of the selected subsamples nor the individual cases.

Our chemical composition analysis shows also that the enrichment from a more evolved stellar companion (Sackmann \& Boothroyd 1999) mechanism is not common among Li-rich giants. Of the 11 stars with significant Li-overabundance, only one, TYC 3314-01371-1 is a spectroscopic binary and shows a very modest oxygen overabundance.

We have also explored the possibility that the lithium abundances might be associated to engulfment of companions that would have a consequence of a mass loss increase. Engulfing low-mass companions is a standard consequence of the increasing radius of the star that climbs the RGB and of tidal interactions (Villaver \& Livio 2009). As this effect would most likely be shown as an infrared excess, we have to search for them using the 2MASS, WISE, and IRAS catalogs, and we do not find any strong evidence in the data of this being the case. None of the 11 Li-rich giants shows $K$-WISE[12 $\mu \mathrm{m}]$ excess. In the de La Reza et al. (1997) diagram only one of Li-rich stars (TYC 3917-1107-1) is present in the high IR excess region II. Of the 78 giants with IRAS data available, only ten with detected $\mathrm{Li}$ $(16 \pm 5 \%)$ belong to the region II, where moderate IR excess is expected. That fraction is lower than in the total sample $(25 \pm 2 \%)$ and we can state that giants with detected $\mathrm{Li}$ or overabundance do not show a trace of a more intense mass loss than giants with no detected $\mathrm{Li}$ in general.
Engulfment has been explored to explain the existence of fast-rotating giants (Carlberg et al. 2009), as the orbital angular momentum of planet might be transferred to a star, which speeds up its rotation. We have identified, however, only one Li-rich fast rotator among Li-rich stars (TYC 1058-02865-1, $v \sin i=$ $6 \mathrm{~km} \mathrm{~s}^{-1}$ ) and another three with $v \sin i>10 \mathrm{~km} \mathrm{~s}^{-1}$, which are classified as Li-poor stars. Our statistical analysis suggests that Li-rich, Li-detectable, and Li-poor stars have identical rotational velocities compared to the rest of stars in sample. The differences found in velocities are marginal, they do not exceed the $1 \mathrm{~km} \mathrm{~s}^{-1}$ level. We did not confirm the relation of $\mathrm{Li}$ content with stellar rotation.

More interesting is the combination of spectroscopic analysis and radial velocity measurements obtained within PTPS. Fiour of 11 stars in the most Li-rich sample, including two the super-Li giants, present low level RV variations pointing to planetary or brown dwarf-mass companions. The giant TYC 330400090-1 has been shown to have a planetary mass companion in a very eccentric orbit by Adamów et al. (2012), who suggest that the high Li content in this giant is due to engulfment of a hypothetical second planet. The object TYC 3917-01107-1 (Niedzielski, in prep.) is another example of Li-rich giant with a planetary system. The other two Li-rich giants also display $\mathrm{RV}$ variations suggesting planetary/brown dwarf companions. In all, our data suggests that the presence of companions is an important factor in the enrichment processes. It may stimulate the $\mathrm{Li}$ production processes through tidal interactions, or by the engulfment of planets or brown dwarf. However, given the lack of evidence of enhanced mass-loss or rotation in Li-rich giants, the mechanism of chemical enrichment in the course of engulfment certainly requires rethinking. In principle the Li overabundance in giants may be connected with the hypothesis by Ramírez et al. (2012) that planets may slow down the Li depletion process in dwarfs located in the so-called "lithium desert" but how that process affects giants remains unclear.

The other seven Li-rich giants present a more standard population with one spectroscopic binary, one star with low amplitude RV variations, and five apparently single stars. The binary frequency in the small sample is $29 \pm 17 \%$ and agrees with a general binary frequency among red giants of $21-30 \%$ (Mermilliod \& Mayor 1992; Gunn \& Griffin 1979). The nature of Li enrichment of those six giants is that they has most likely not completed FDU dilution yet. Statistical analysis pointing to higher masses and effective temperatures for Li-detectable and Li-rich stars support this conclusion. Massive stars spend less time on MS than solar-masses objects; hence, their depletion in this evolutionary stage is not as severe. Observed $A(\mathrm{Li})$ abundances for 2.5-3 $M_{\odot}$ MS stars show Li abundances up to meteoritic level. Their location at the base or in lower RGB with $A(\mathrm{Li}) \sim 1.4-1.5$ is then not surprising.

Only one star, TYC 0405-01700-1, seems to present characteristics attributed to several Li-enrichment processed at the same time. It has a stellar companion, seems to be fast rotator, and may be reveling some kind of chromospheric activity. It is also located in the LFB region, and it is the only star that shows low IR excess. It shows $\mathrm{O}$ overabundance: its source is probably not accretion of supernova remnants but a transfer from a stellar companion. Although this star is a very difficult case to spectroscopic studies, it is certainly a very interesting object. In his work, Denissenkov (2012) suggests that Li rich stars reveal characteristics similar to RS CVn stars. It may be indeed the case for TYC 0405-01700-1.

Acknowledgements. We thank Dr. Nikolai Piskunov and Dr. Jeff Valenti for making SME available for us. We thank the HET and IAC resident astronomers 
and telescope operators for support. We thank the anonymous referee for comments that resulted in substantial improvement of this paper. M.A., A.N. and G.N. were supported in part by the Polish Ministry of Science and Higher Education grant No. N203 510938 and by the Polish National Science Centre grant No. UMO-2012/07/B/ST9/04415. M.A. is also supported by the Polish National Science Centre grant No. UMO-2012/05/N/ST9/03836. G.N. is also supported by The Faculty of Physics, Astronomy and Informatics of the Nicolaus Copernicus University grant No. 1627-A. E.V. work was supported by the Spanish Ministerio de Ciencia e Innovacion (MICINN), Plan Nacional de Astronomia y Astrofísica, under grant AYA2010-20630 and by the Marie Curie grant FP7-People-RG268111. A.W. was supported by the NASA grant NNX09AB36G. The HET is a joint project of the University of Texas at Austin, the Pennsylvania State University, Stanford University, LudwigMaximilians-Universität München, and Georg-August-Universität Göttingen. The HET is named in honor of its principal benefactors, William P. Hobby and Robert E. Eberly. The Center for Exoplanets and Habitable Worlds is supported by the Pennsylvania State University, the Eberly College of Science, and the Pennsylvania Space Grant Consortium. This research has made use of the SIMBAD database, operated at CDS (Strasbourg, France) and NASA's Astrophysics Data System Bibliographic Services. This research has made use of the NASA/IPAC Infrared Science Archive, which is operated by the Jet Propulsion Laboratory, California Institute of Technology, under contract with the National Aeronautics and Space Administration.

\section{References}

Adamów, M., Niedzielski, A., Villaver, E., Nowak, G., \& Wolszczan, A. 2012, ApJ, 754, L15

Alexander, J. B. 1967, The Observatory, 87, 238

Anthony-Twarog, B. J., Deliyannis, C. P., Rich, E., \& Twarog, B. A. 2013, ApJ, 767, L19

Arnett, W. D. 1978, Mem. Soc. Astron. It., 49, 431

Asplund, M., Grevesse, N., Sauval, A. J., \& Scott, P. 2009, ARA\&A, 47, 481

Bertelli, G., Girardi, L., Marigo, P., \& Nasi, E. 2008, A\&A, 484, 815

Brown, J. A., Sneden, C., Lambert, D. L., \& Dutchover, Jr., E. 1989, ApJS, 71, 293

Busso, M., Wasserburg, G. J., Nollett, K. M., \& Calandra, A. 2007, ApJ, 671, 802

Cameron, A. G. W., \& Fowler, W. A. 1971, ApJ, 164, 111

Cannon, R. D. 1970, MNRAS, 150, 111

Carlberg, J. K., Majewski, S. R., \& Arras, P. 2009, ApJ, 700, 832

Carlberg, J. K., Smith, V. V., Cunha, K., Majewski, S. R., \& Rood, R. T. 2010, ApJ, 723, L103

Carlberg, J. K., Majewski, S. R., Patterson, R. J., et al. 2011, ApJ, 732, 39

Carlberg, J. K., Cunha, K., Smith, V. V., \& Majewski, S. R. 2012, ApJ, 757, 109

Carlsson, M., Rutten, R. J., Bruls, J. H. M. J., \& Shchukina, N. G. 1994, A\&A, 288,860

Carney, B. W., Gray, D. F., Yong, D., et al. 2008, AJ, 135, 892

Charbonnel, C., \& Balachandran, S. C. 2000, A\&A, 359, 563

Charbonnel, C., \& Zahn, J.-P. 2007, A\&A, 467, L15

Cosentino, R., Lovis, C., Pepe, F., et al. 2012, in SPIE Conf. Ser., 8446

de La Reza, R., Drake, N. A., da Silva, L., Torres, C. A. O., \& Martin, E. L. 1997, ApJ, 482, L77

de Medeiros, J. R., \& Mayor, M. 1999, A\&AS, 139, 433

Denissenkov, P. A. 2012, ApJ, 753, L3

Denissenkov, P. A., Pinsonneault, M., \& MacGregor, K. B. 2009, ApJ, 696, 1823

Drake, N. A., de la Reza, R., da Silva, L., \& Lambert, D. L. 2002, AJ, 123, 2703

Eggleton, P. P., Dearborn, D. S. P., \& Lattanzio, J. C. 2008, ApJ, 677, 581

Fekel, F. C. 1997, PASP, 109, 514

Frandsen, S., \& Lindberg, B. 1999, in Astrophysics with the NOT, eds. H. Karttunen, \& V. Piirola, 71
Ghezzi, L., Cunha, K., Smith, V. V., \& de la Reza, R. 2010, ApJ, 724, 154 Gonzalez, O. A., Zoccali, M., Monaco, L., et al. 2009, A\&A, 508, 289 Gray, D. F. 1989, ApJ, 347, 1021

Gunn, J. E., \& Griffin, R. F. 1979, AJ, 84, 752

Haakonsen, C. B., \& Rutledge, R. E. 2009, ApJS, 184, 138

Hekker, S., \& Meléndez, J. 2007, A\&A, 475, 1003

Iben, I. 2013, Stellar Evolution Physics (Cambridge University Press) Iben, Jr., I. 1967, ApJ, 147, 624

Ibukiyama, A., \& Arimoto, N. 2002, A\&A, 394, 927

Jimenez, R., Flynn, C., \& Kotoneva, E. 1998, MNRAS, 299, 515

Johnson, D. R. H., \& Soderblom, D. R. 1987, AJ, 93, 864

Kiraga, M. 2012, Acta Astron., 62, 67

Kumar, Y. B., Reddy, B. E., \& Lambert, D. L. 2011, ApJ, 730, L12

Kupka, F., Piskunov, N., Ryabchikova, T. A., Stempels, H. C., \& Weiss, W. W. 1999, A\&AS, 138, 119

Kurucz, R. 1993, ATLAS9 Stellar Atmosphere Programs and $2 \mathrm{~km} \mathrm{~s}^{-1}$ grid, Kurucz CD-ROM No. 13 (Cambridge, Mass.: Smithsonian Astrophysical Observatory), 13

Lebzelter, T., Uttenthaler, S., Busso, M., Schultheis, M., \& Aringer, B. 2012, A\&A, 538, A36

Lind, K., Asplund, M., \& Barklem, P. S. 2009, A\&A, 503, 541

Luck, R. E., \& Heiter, U. 2007, AJ, 133, 2464

Maldonado, J., Villaver, E., \& Eiroa, C. 2013, A\&A, 554, A84

Mallik, S. V. 1999, A\&A, 352, 495

Martell, S. L., \& Shetrone, M. D. 2013, MNRAS, 430, 611

Massarotti, A., Latham, D. W., Stefanik, R. P., \& Fogel, J. 2008, AJ, 135, 209

McWilliam, A. 1997, ARA\&A, 35, 503

Mermilliod, J.-C., \& Mayor, M. 1992, in Binaries as Tracers of Star Formation, eds. A. Duquennoy, \& M. Mayor, 183

Neugebauer, G., Habing, H. J., van Duinen, R., et al. 1984, ApJ, 278, L1

Niedzielski, A., \& Wolszczan, A. 2008, in Extreme Solar Systems, eds. D. Fischer, F. A. Rasio, S. E. Thorsett, \& A. Wolszczan, ASP Conf. Ser., 398, 71

Niedzielski, A., Konacki, M., Wolszczan, A., et al. 2007, ApJ, 669, 1354

Nowak, G. 2012, Ph.D. Thesis, Nicolaus Copernicus Univ., Toruń, Poland

Nowak, G., Niedzielski, A., Wolszczan, A., Adamów, M., \& Maciejewski, G. 2013, ApJ, 770, 53

Ramírez, I., \& Allende Prieto, C. 2011, ApJ, 743, 135

Ramírez, I., Allende Prieto, C., \& Lambert, D. L. 2007, A\&A, 465, 271

Ramírez, I., Fish, J. R., Lambert, D. L., \& Allende Prieto, C. 2012, ApJ, 756, 46

Ramsey, L. W., Adams, M. T., Barnes, T. G., et al. 1998, in SPIE Conf. Ser. 3352, ed. L. M. Stepp, 34

Raskin, G., van Winckel, H., Hensberge, H., et al. 2011, A\&A, 526, A69

Reddy, B. E., \& Lambert, D. L. 2005, AJ, 129, 2831

Reddy, B. E., Lambert, D. L., Hrivnak, B. J., \& Bakker, E. J. 2002, AJ, 123, 1993

Sackmann, I.-J., \& Boothroyd, A. I. 1999, ApJ, 510, 217

Shetrone, M., Cornell, M. E., Fowler, J. R., et al. 2007, PASP, 119, 556

Siess, L., \& Livio, M. 1999, MNRAS, 308, 1133

Smith, V. V., Cunha, K., Shetrone, M. D., et al. 2013, ApJ, 765, 16

Spite, M., Andrievsky, S. M., Spite, F., et al. 2012, A\&A, 541, A143

Student. 1908, Biometrika, 6, 1

Stumpff, P. 1980, A\&AS, 41, 1

Tautvaisiene, G., \& Puzeras, E. 2009, in IAU Symp. 254, eds. J. Andersen, B. M. Nordströara, \& J. Bland-Hawthorn, 75

Tull, R. G. 1998, in SPIE Conf. Ser. 3355, eds. S. D’Odorico, 387

Valenti, J. A., \& Piskunov, N. 1996, A\&AS, 118, 595

van Leeuwen, F. 2007, A\&A, 474, 653

Villaver, E., \& Livio, M. 2009, ApJ, 705, L81

Woosley, S. E., \& Weaver, T. A. 1995, ApJS, 101, 181

Wright, E. L., Eisenhardt, P. R. M., Mainzer, A. K., et al. 2010, AJ, 140, 1868

Zieliński, P., Niedzielski, A., Wolszczan, A., Adamów, M., \& Nowak, G. 2012, A\&A, 547, A91 